\title{
UICN
}

\section{Reconnaissance et signalement des autres mesures de conservation efficaces par zone}

Groupe de travail sur les AMCE de la Commission mondiale des aires protégées de l'UICN
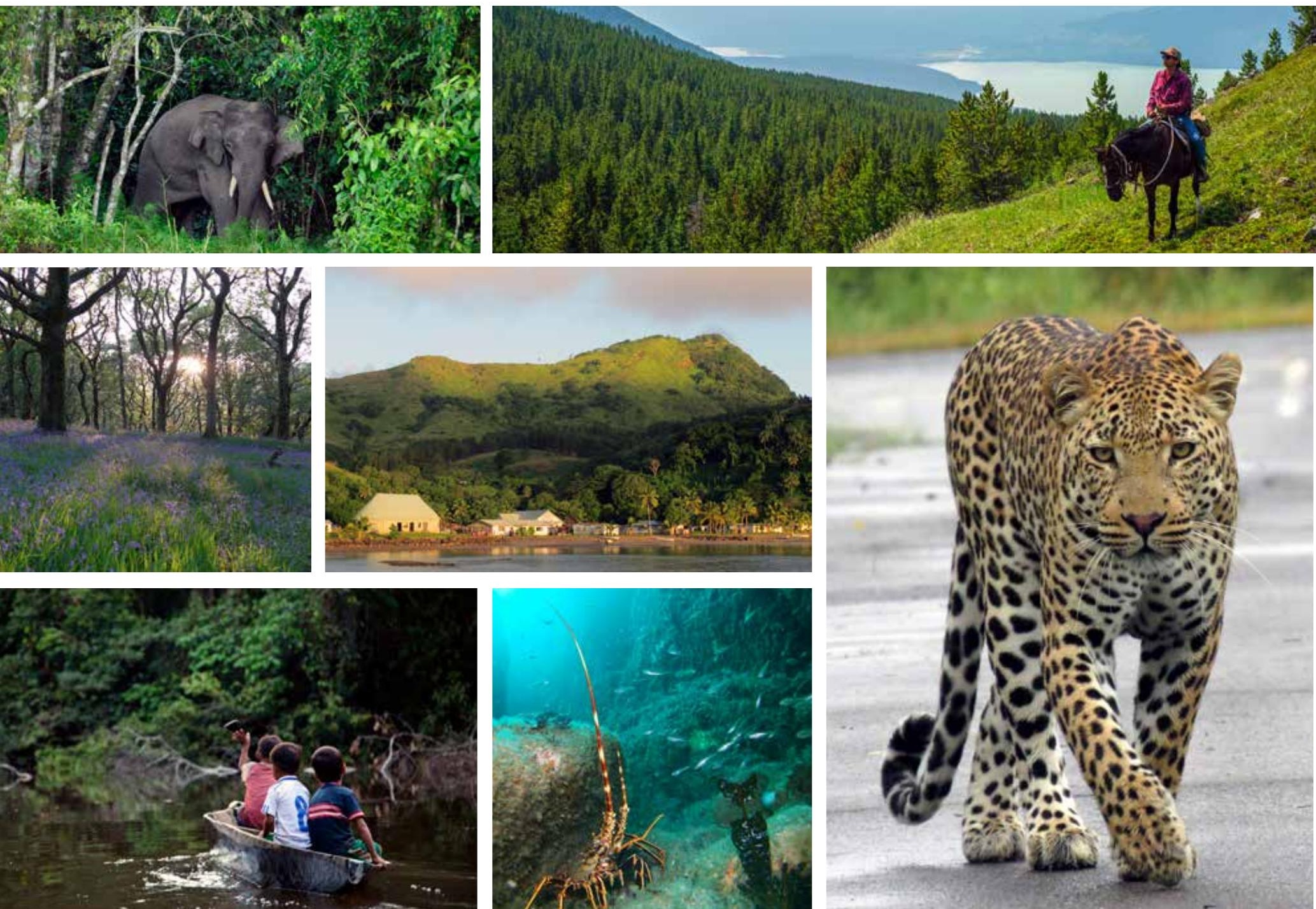

Rapport technique des aires protégées, Série $n^{\circ} 3$

5 CMAP 



\section{Reconnaissance et signalement des autres mesures de conservation efficaces par zone}

Groupe de travail sur les AMCE de la Commission mondiale des aires protégées de l'UICN 
La terminologie géographique employée dans cet ouvrage, de même que sa présentation, ne sont en aucune manière l'expression d'une opinion quelconque de la part de l'UICN ou des autres organisations concernées sur le statut juridique ou l'autorité de quelque pays, territoire ou région que ce soit, ou sur la délimitation de ses frontières.

Les opinions exprimées dans cette publication ne reflètent pas nécessairement celles de l'UICN ou des autres organisations concernées.

Tous les exemples sont fournis conformément à leur état et statut à la date de publication.

L'UICN et les autres organisations concernées rejettent toute responsabilité en cas d'erreurs ou d'omissions intervenues lors de la traduction en français de ce document dont la version originale est en anglais. En cas de divergences, veuillez vous référer à l'édition originale : Recognising and reporting other effective area-based conservation measures. Gland, Suisse: UICN. DOI : https://doi. org/10.2305/IUCN.CH.2010.PATRS.3.en

Publié par : UICN, Gland, Suisse

Droits d'auteur : (c) 2019 UICN, Union internationale pour la conservation de la nature et de ses ressources.

(C) 2020 UICN, Union internationale pour la conservation de la nature et de ses ressources, pour la traduction française

La reproduction de cette publication à des fins non commerciales, notamment éducatives, est permise sans autorisation écrite préalable des détenteurs des droits d'auteur à condition que la source soit dûment citée. La reproduction de cette publication à des fins commerciales, notamment en vue de la vente, est interdite sans autorisation écrite préalable des détenteurs des droits d'auteur.

Citation : Groupe de travail sur les AMCE de la CMAP-UICN (2020). Reconnaissance et signalement des autres mesures de conservation efficaces par zone. Gland, Suisse : UICN.

ISBN: 978-2-8317-2067-8 (PDF)

DOI: https://doi.org/10.2305/IUCN.CH.2019.PATRS.3.fr

Photos de couverture : Haut (gauche) : 6. Éléphant pygmée se déplaçant le long d'une rivière dans une zone gérée par la communauté, à proximité du village d'Abai dans le site Ramsar des zones humides du bas Kinabatangan-Segama, Sabah (Malaisie). () Harry Jonas. Haut (droite) : William Myers, membre de la Première Nation Yunesit'in, l'une des six communautés de la Nation Tsilhqot'in, traversant à cheval les montagnes du Dasiqox Tribal Park (Colombie-Britannique, Canada). () Jeremy Williams. Bas (droite) : Léopard marchant le long de la piste de la base aérienne de Hoedspruit, laquelle soutient une aire de conservation de plus de 2000 ha autour de la base, utilisée pour les formations et en tant que zone tampon avec les civils (Hoedspruit, Afrique du Sud). () Hoedspruit Airforce Base. Bas (milieu) : Site d'épaves historiques dans les îles Scilly (Royaume-Uni), dans lequel toute activité est exclue, ce qui procure un environnement non perturbé permettant à la faune sauvage marine de s'épanouir. (C) Dan Laffoley. Bas (gauche) : Des enfants de la région de Loreto (Pérou) découvrent les environs dans une concession de conservation. (c) Bruno Monteferri. Centre (gauche) : La Réserve de biosphère de Dyfi (centre du Pays de Galles) abrite un ensemble d'aires protégées gérées de manière privée ou gouvernementale, ainsi que des terrains privés, servant principalement à l'élevage de bovins et d'ovins. Ces terrains comprennent des zones à forte valeur de biodiversité, comme les forêts anciennes. Au-delà de la reconnaissance de ces valeurs par la désignation « biosphère ", une initiative de plusieurs millions de livres sterling est dédiée à la restauration du processus naturel dans la vallée de Dyfi, laquelle englobe des montagnes, des pâturages, des zones boisées, des tourbières, un large estuaire et une zone marine dotée de vastes systèmes plages/ dunes. Ciblant au moins 10000 hectares de terres et 28400 ha en mer, la superficie totale couvre probablement de multiples AMCE potentielles. (c) Equilibrium Research. Centre : Coucher de soleil sur le village de Tovu (île de Totoya, Fidji), où les responsables de la communauté intègrent des approches portant sur les aires marines gérées localement, avec une planification à l'échelle du paysage marin. (C) Stacy Jupiter. Dos : Un jeune membre d'une famille Bajau naviguant près d'un récif en début de soirée (Sabah, Malaisie). () Harry Jonas.

Traduction : Alexa Dubreuil-Storer (IDFP Translation Services), Royaume-Uni

Conception et mise en page : Miller Design

Disponible auprès de : Union internationale pour la conservation de la nature (UICN)

Programme global des aires protégées

Rue Mauverney 28

1196 Gland, Suisse

wcpa@iucn.org

www.iucn.org/resources/publications 


\section{Table des matières}

Avant-propos IV IV

Résumé $\quad v$

Remerciements vi

Glossaire viii

Acronymes $\quad x$

Liste des encadrés, tableaux et figures $\quad x$

1. Introduction $\quad 1$

2. Définition et caractéristiques 3

3. Recensement des autres mesures de conservation efficaces par $\begin{array}{ll}\text { zone en pratique } & 8\end{array}$

4. Suivi des autres mesures de conservation efficaces par zone et établissement de rapports à leur sujet

Références

Annexe I - Relation entre les Objectifs d'Aichi et l'Objectif $11 \quad 16$

Annexe II - Aide à la décision : L'Objectif 11 est-il le plus pertinent des

Objectifs d'Aichi pour évaluer une mesure de conservation? 18

Annexe III - Base de données mondiale sur les aires protégées 20 


\section{Avant-propos}

De nombreuses zones situées en dehors des réseaux d'aires protégées nationaux et régionaux contribuent aussi à la conservation in situ efficace de la biodiversité. Bien reconnaître et soutenir ces zones, et établir des rapports pertinents à leur sujet est de plus en plus important dans le contexte de l'appauvrissement de la biodiversité et du changement climatique. Les Parties à la Convention sur la diversité biologique (CDB) ont reconnu au début du Plan stratégique (2011-2020) de la CDB que "d'autres mesures de conservation efficaces par zone » (AMCE) offrent une réelle occasion d'atteindre cet objectif.

L'avis technique donné par le Groupe de travail sur les autres mesures de conservation efficaces par zone (AMCE) de la Commission mondiale des aires protégées (CMAP) de I'UICN a contribué à l'adoption, lors de la 14e Conférence des Parties à la $\mathrm{CDB}$, d'une définition, de principes directeurs, de caractéristiques communes et de critères de recensement des AMCE (Décision 14/8 de la CDB). Le monde a maintenant l'occasion de mieux reconnaître la conservation de facto qui a lieu en dehors des aires protégées actuellement désignées, et qui est réalisée par différents types d'acteurs, y compris les Populations autochtones, les communautés locales, le secteur privé et les agences gouvernementales.

La reconnaissance appropriée des AMCE offre l'occasion de faire participer et de soutenir les détenteurs de droits et les parties prenantes, et de promouvoir des partenariats plus équitables dans les efforts mondiaux de conservation, en mettant en évidence la diversité des contributions en matière de conservation à l'échelle mondiale. Ce faisant, les AMCE contribueront à la conservation de la biodiversité de bien des façons, telles que : la préservation d'importants écosystèmes, habitats et corridors fauniques représentatifs ; le soutien à la récupération des espèces menacées
; le maintien des fonctions des écosystèmes et la sécurisation des services écosystémiques ; l'amélioration de la résilience face aux menaces ; et la contribution à une meilleure gestion et restauration des zones qui pourraient être utiles pour soutenir la conservation in situ de la biodiversité à long terme. Les AMCE peuvent contribuer à des réseaux écologiquement représentatifs et bien reliés d'aires protégées et conservées, et intégrées dans l'ensemble du paysage terrestre et marin.

Comme pour tout «nouveau » cadre, il faudra probablement une interprétation et une mise en œuvre continues. Le maintien de la pleine valeur des AMCE pour promouvoir une conservation efficace nécessitera probablement des efforts considérables afin de renforcer les capacités aux niveaux national et régional pour identifier, surveiller et maintenir leurs valeurs en termes de biodiversité. Les AMCE offrent une occasion intéressante de reconnaître et d'élargir le périmètre de conservation, dans le cadre d'un ensemble de régimes de gouvernance et de gestion, comme l'envisage l'Objectif 11 d'Aichi. Un défi clé sera de savoir comment reconnaître et soutenir ces zones entièrement conservées tout en respectant les obligations nationales pour une gestion plus durable des activités de production, y compris dans les secteurs de la sylviculture, de l'agriculture et de la pêche industrielles qui ne répondent pas aux critères des $\mathrm{AMCE}$, mais qui peuvent également contribuer aux Objectifs de développement durable (ODD).

Alors que les Parties à la Convention délibèrent sur le Cadre mondial de la biodiversité pour l'après-2020, ces lignes directrices continueront d'apporter une contribution importante pour s'assurer que les AMCE sont correctement reconnues et contribuent à la fois aux objectifs de couverture et à la représentation écologique par le biais de « réseaux d'aires protégées et d'autres mesures de conservation efficaces par zone ».

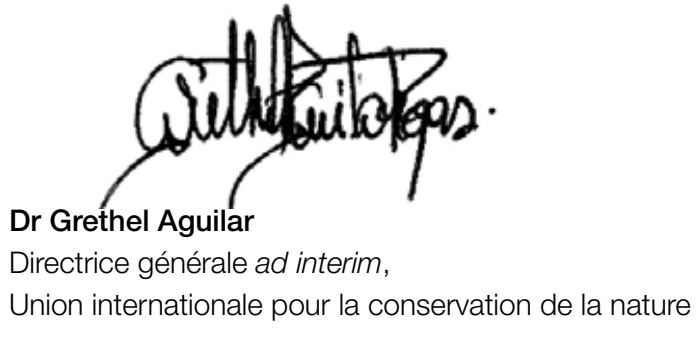

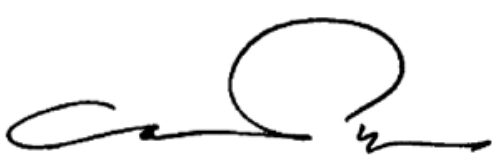

Dr. Cristiana Pașca Palmer

Secrétaire exécutive,

Convention sur la diversité biologique 


\section{Résumé}

Le Plan stratégique pour la biodiversité 2011-2020, adopté à Nagoya, fournit un cadre pour la mise en œuvre efficace de la Convention sur la diversité biologique (CDB) avec 20 objectifs couvrant la période 2011-2020. L'Objectif 11 d'Aichi indique que la conservation se fera au moyen de réseaux écologiquement représentatifs et bien reliés d'aires protégées gérées efficacement et équitablement et d'autres mesures de conservation efficaces par zone. Même s'il existait déjà des définitions et des critères clairs pour les aires protégées, ce n'était pas le cas des « autres mesures de conservation efficaces par zone ».

En novembre 2018, cette situation a été rectifiée lorsque les Parties à la CDB ont adopté, à l'occasion de la 14e Conférence des Parties, une définition du terme " autre mesure de conservation efficace par zone » (AMCE), ainsi que des principes directeurs, des caractéristiques communes et des critères de recensement d'AMCE (CBD/COP/DEC/14/8). La Décision 14/8 définit une AMCE comme suit :

Une zone géographiquement délimitée, autre qu'une aire protégée, qui est réglementée et gérée de façon à obtenir des résultats positifs et durables à long terme pour la conservation in situ de la diversité biologique, y compris des fonctions et services écosystémiques connexes et, le cas échéant, des valeurs culturelles, spirituelles, socioéconomiques et d'autres valeurs pertinentes localement.

Même si les aires protégées doivent avoir un objectif de conservation primaire, cela n'est pas nécessaire pour les AMCE. Les AMCE peuvent être gérées pour de nombreux objectifs différents, mais elles doivent aboutir à une conservation efficace. Elles peuvent être gérées en prenant la conservation comme objectif primaire ou secondaire, ou bien la conservation à long terme peut être simplement le résultat dérivé des activités de gestion.

La reconnaissance des AMCE offre une réelle occasion de mieux reconnaître la conservation efficace à long terme de facto qui a lieu en dehors des aires protégées actuellement désignées, dans le cadre d'un ensemble de régimes de gouvernance et de gestion, et qui est réalisée par différents types d'acteurs, y compris les populations autochtones, les communautés locales, le secteur privé et les agences gouvernementales. Les AMCE peuvent contribuer à des réseaux de conservation écologiquement représentatifs et bien reliés, intégrés dans l'ensemble du paysage terrestre et marin, et ce faisant, générer de multiples résultats positifs pour la conservation. Par exemple :

- La préservation des écosystèmes, habitats et corridors fauniques importants ;

- Le soutien à la récupération des espèces menacées ;

- Le maintien des fonctions des écosystèmes et la sécurisation des services écosystémiques ;

- L'amélioration de la résilience face aux menaces ; et

- La préservation et la mise en relation des vestiges d'écosystèmes fragmentés dans les paysages terrestres dégradés.

Le Groupe de travail sur les AMCE de la CMAP a produit ce Rapport technique pour aider les Parties à interpréter et à rendre opérationnelle la Décision 14/8, et pour commencer à élaborer un ensemble de bonnes pratiques en matière de reconnaissance des AMCE et d'établissement de rapports à leur sujet. II a été conçu pour une application à diverses échelles, par exemple pour comprendre si une zone individuelle est une AMCE, ou pour établir des rapports statistiques sur les AMCE au niveau national et mondial afin d'évaluer les progrès enregistrés pour atteindre les objectifs de conservation.

La $1^{\text {re }}$ partie présente le contexte du terme « autre mesure de conservation efficace par zone » et un aperçu du processus qui a conduit à la Décision 14/8 de la CDB.

La $2^{\mathbf{e}}$ partie décrit la définition d'une AMCE et fournit des explications claires sur chaque élément de la définition et les critères.

La $3^{\mathbf{e}}$ partie présente un outil de présélection simple qui peut être utilisé pour recenser les "AMCE candidates ", et elle fournit une liste indicative d'exemples d'AMCE potentielles et de zones peu susceptibles de répondre aux critères. II est important que cette présélection et les évaluations ultérieures soient effectuées site par site afin de s'assurer que les zones répondent aux critères visant une conservation efficace à long terme.

La 4e partie décrit les processus relatifs au suivi des AMCE et à l'établissement de rapports à leur sujet, en mettant l'accent sur les bases de données mondiales de Protected Planet, gérées par le Centre mondial de surveillance continue de la conservation de la nature du PNUE. 


\section{Remerciements}

\begin{abstract}
Les présentes lignes directrices ont été élaborées par le Groupe de travail sur les autres mesures de conservation efficaces par zone (AMCE) de la Commission mondiale des aires protégées de l'UICN, créé en 2015. Le Groupe de travail a travaillé pendant trois ans en soutien à un processus de la Convention sur la diversité biologique (CDB), aboutissant à la Décision 14/8 de la CDB sur les « aires protégées et autres mesures de conservation efficaces par zone ", adoptée par 196 Parties lors de la 14e Conférence des Parties (novembre 2018). Cet avis technique concerne directement la Décision 14/8 de la CDB sur la reconnaissance des autres mesures de conservation efficaces par zone (AMCE) et l'établissement de rapports sur les AMCE, lesquelles sont parfois appelées « aires conservées ».
\end{abstract}

\section{Le Groupe de travail était coprésidé par Kathy MacKinnon (président de la CMAP, Royaume-Uni) et Harry Jonas} (Royaume-Uni/Malaisie) et réunissait plus de 120 membres experts. Ces lignes directrices ont été éditées par les coprésidents et un groupe de rédaction composé de Nigel Dudley (Royaume-Uni), Marc Hockings (Australie), Dan Laffoley (RoyaumeUni), David MacKinnon (Canada), Trevor Sandwith (Afrique du Sud) et Stephen Woodley (Canada).

\section{Quatre ateliers d'experts du Groupe de travail ont été} organisés : à Cambridge au Royaume-Uni (janvier 2016), à Vilm en Allemagne (juillet 2016 et juillet 2019) et à Vancouver au Canada (février 2017). La CMAP-UICN tient à remercier l'Agence fédérale allemande pour la conservation de la nature (BfN), le Département fédéral de l'environnement de la Suisse, SwedBio et la Société pour la nature et les parcs du Canada (SNAP) pour leur contribution financière aux travaux du Groupe de travail. Nous remercions le personnel du Centre mondial de surveillance continue de la conservation de la nature (PNUE-WCMC) à Cambridge (Royaume-Uni), Gisela Stolpe et Bettina Ohnesorge (BfN) à Vilm (Allemagne) et Sabine Jessen (SNAP) à Vancouver (Canada) de leur soutien logistique pour l'organisation et la facilitation de ces réunions. Nous remercions également Sarat Gidda et ses collègues du Secrétariat de la CDB pour leur participation active tout au long du processus, notamment en coorganisant plusieurs événements en marge des réunions des Parties.

\section{Lors du processus d'élaboration, les membres du Groupe de travail et d'autres experts ont fourni de précieux commentaires, renseignements et études de cas. Nous exprimons notre reconnaissance envers les personnes suivantes pour leur contribution (les personnes ayant contribué aux études de cas sont signalées par un astérisque) : Agnes Agama (Malaisie), Tundi Agardy (États-Unis), Khaled Allam Harhash (Égypte), Helena Alvez-Pinto (Brésil), Thora Amend* (Allemagne), Michele Andrianarisata* (Madagascar), Ny Aina Andrianarivelo* (Madagascar), Ludi Apin (Malaisie), Alexandra Areiza (Colombie), Clarissa Arida (Philippines), Peter Auster (États-Unis), Ghanimat Azhdari (Iran), Tim Badman (Royaume-Uni/Suisse), Megan Barnes (Australie), Juan Bezaury-Creel (Mexique), Seema Bhatt (Inde), Dominique Bikaba (République démocratique du Congo), Heather Bingham* (Royaume-Uni), Grazia Borrini Feyerabend* (Italie/}

Suisse), Peter Bridgewater (Australie), Johnny Briggs (RoyaumeUni), Thomas Brooks (Royaume-Uni/Suisse), Jessica Brown* (États-Unis), Jens Bruggemann* (Allemagne), Neil Burgess (Royaume-Uni), Catie Burlando (Italie), Stuart Butchart (RoyaumeUni), Pete Chaniotis (Royaume-Uni), Maria Elfi Chávez (Colombie), Gladman Chibememe (Zimbabwe), Christie Chute* (Canada), Pepe Clark (Australie/Royaume-Uni), Peter Cochrane (Australie), Laura Cornick (Royaume-Uni), Mark Costello (Nouvelle-Zélande), Mason Croft (États-Unis), Adrian Davey (Australie), Mimi D’lorio (ÉtatsUnis), Pablo Dominguez (Espagne), Paul Donald* (Royaume-Uni), Alkaly Doumbouya* (Guinée), Lisa Duarte (États-Unis), Steve Edwards (Royaume-Uni/Suisse), Hany El Shaer (Égypte), Cristina Eghenter* (Italie), Shahul Faizi Hameed (Inde), Edgar Fernández (Costa Rica), Gregor Fischenich (Allemagne), Vin Fleming (Royaume-Uni), Amelia Fowles (Australie), Christine Franklin (États-Unis), Sandra Galán (Colombie), Delfin Ganapin (Philippines), Carolina Garcia Imhof (Colombie), Sonali Ghosh (Inde), Rachel Golden Kroner (États-Unis), Hugh Govan (Royaume-Uni/Fidji), Stephen Grady (Royaume-Uni), Tarsicio Granizo (Équateur), Tarsicio Granizo (Équateur), Ania Grobicki (Afrique du Sud/Suisse), Catalina Gutierrez (Colombie), James Hardcastle (Royaume-Uni/ Suisse), Terence Hay-Edie (Suisse/Thaillande), Yifan (Flora) He (Chine), Robert Hélie (Canada), Ro Hill (Australie), Amber HimesCornell (États-Unis), Marc Hockings (Australie), Elaine Hsiao (Canada), Claudia Ituarte Lima (Mexique), Sabine Jessen* (Canada), Holly Jonas (Canada/Malaisie), Stacy Jupiter (ÉtatsUnis/Fidji), Theodore Karfakis (Grèce), Jennifer Kelleher (Irlande), Kate Kincaid (Canada), Jonathan Kirui (Kenya), Rebecca Klaus (Royaume-Uni), Naomi Kingston* (Irlande/Royaume-Uni), Eskild Kirkegaard (Danemark), Mirjam de Konig (Pays-Bas), Sigrid Kuehnemund (Canada), Barbara Lang (Allemagne/Bénin), Thierry Lefebvre (France), Christopher Lemieux (Canada), Clare Lewis (Royaume-Uni), Harvey Locke (Canada), Julia Miranda Londono (Colombie), Ali Mahamane (Niger), Claudio Maretti (Brésil), Michael Mascia (États-Unis), Lisa McLaughlin (Canada), Daniel Marnewick (Afrique du Sud), Clara Lucía Matallana-Tobón* (Colombie), Pradeep Mehta (Inde), Mehmet Metaj (Albanie), Rossana Merizalde (États-Unis), Amy Milam (États-Unis), Carmen Miranda (Bolivie), Brent Mitchell* (États-Unis), Rahul Mungikar (Inde) Daniel Mwamidi (Kenya), Helen Newing (Royaume-Uni), Onkemetse Nteta* (Afrique du Sud), Melissa Jane Nursey-Bray (Australie), Aboubacar Oularé (Guinée), Gisela Paredes (Colombie), Roberto Pereyra Lobos (Argentine), Jacques Perron* (Canada), Dimitra Petza (Grèce/Italie), Hugh Possingham (Australie), Jeff Pradel (Pérou), Madhu Rao (Inde/Royaume-Uni/Singapour), Andrew Rhodes* (Mexique), Ryan Richards (États-Unis), Danielle Ryan (Australie), Marina Rosales (Pérou), Carlos Saavedra (Colombie), Yoav Sagi (Israël), Marcela Santamaria (Colombie), Elsa Sattout (Liban), Klaus Schmitt* (Allemagne), Faizi Shahul Hameed (Inde), Sushma Shresthma (Népal/États-Unis) Joanna Smith (Canada), Dermot Smyth (Australie), Clara Solano (Colombie), Mark Spalding (RoyaumeUni), Candice Stevens (Afrique du Sud), Todd Stevenson (ÉtatsUnis), Mavra Stithou (Grèce), Sue Stolton (Royaume-Uni), Teki Surayya (Inde), Kim Taylor Thompson* (Canada), Parfait Tchuenfo (Cameroun), Anteneh Tesfaw (Éthiopie), David Thomas (RoyaumeUni), Ted Trzyna (États-Unis), Agus Utomo* (Indonésie), Bas Verschuuren (Pays-Bas), Francis Vorhies (Royaume-Uni), Agus 
Budi Utomo* (Indonésie), John Waithaka* (Kenya), Sonam Wangchuk $^{\star}$ (Bhoutan), James Watson (Australie), Sue Wells (Royaume-Uni), Sheila Wertz-Kanounnikoff (Allemagne), Tara Whitty (États-Unis), Hesti Widodo* (Indonésie), Ryan Wilkie (Royaume-Uni/Danemark), James Williams (Royaume-Uni), Linda Wong (Chine), Dale Wright (Afrique du Sud), Kim Sander Wright* (Canada), Llewellyn Young* (Hong Kong/Suisse), Hag Young Heo (République de Corée) et Jinfeng Zhou (Chine).

\section{D'autres personnes ont aimablement apporté des} contributions ou des études de cas ; parmi elles : Simon

Albert (Australie), Eugenio Barrios (Mexique), Alexandra Barron (Canada), Nicole Bendsen (Allemagne), Bastian Bertzky (Afrique du Sud/Italie), Caroline Butler (Canada), Tony Charles (Canada), Sushila Chatterjee Nepali (Népal), Roger Crofts (Royaume-Uni), Terence Dacles (Philippines), Steve Diggon (Canada), William Dunbar (États-Unis/Japon), Kim Dunn (Canada), Jessica Elliott (Canada), Ninel Escobar (Mexique), Roman Eyholzer (Suisse), Fred Ford (Australie), Robyn Forrest (Canada), Kim Friedman (Australie/ Italie), Mervi Heinonen (Finlande), Erich Hoyt (États-Unis/RoyaumeUni), Olaf Jensen (Canada), François Lengrand (France), Satnam Manhas (Canada), Joe McCarter (Nouvelle-Zélande), Martine Maron (Canada), Chris McDougall (Canada), Günter Mühlbauer (Allemagne), Mariana Zareth Nava Lopez (Mexique), Saw Tun Khaing (Myanmar), Linda Nowlan (Canada), Shane Orchard (Nouvelle-Zélande), Allison Pritchard (Canada), Ravaka Ranaivoson (Madagascar), Sergio Salinas-Rodríguez (Mexique), Paul Scholte (Allemagne), Archana Sharma (États-Unis), Rebecca Singleton (Royaume-Uni/Canada), Charlotta Sörqvist (Suède), Bruce Stewart (Canada), Gary Tabor (États-Unis), Kaori Tsujita (Japon), Siyu Qin (Chine/États-Unis), Basile Van Havre (Canada), Liette Vasseur (Canada), Scott Wallace (Canada), Bill Wareham (Canada), Gladys Warigia Njoroge (Kenya), Mike Wong (Canada), Alison Woodley (Canada), Edgar Yerena (Venezuela) et Natori Yoji (Japon).

La CMAP remercie également John Waithaka et Lucy Waruingi qui ont organisé un atelier au Kenya sur une version préliminaire des lignes directrices en 2017, ainsi que les personnes suivantes qui ont testé ces orientations sur le terrain aux Bermudes : Alison Copeland, Peter Drew, Dan Laffoley, Jeremy Madeiros, Sarah Manuel, Simieon Massey, Drew Pettit, Joanna Pitt, Philippe Rouja, Mandy Shailer, Robbie Smith, Tammy Trott, Craig Trott et Julie Marshall. La production d'un grand nombre d'études de cas en Colombie a été dirigée par Clara Matallana (Instituto Humboldt), avec le soutien d'Alexandra Areiza, de Maria Elfi Cháves, de Sandra Galán, de Clara Solano et de Marcela Santamaria, et le concours des personnes suivantes : Jony Albeiro Arias, Luis Alimaco, Germán Andrade, Mónica Arroyave, Atanasio Barros, Hermes Carreño, Claudia Céspedes, Stephanie Gailer, Daniel Garavito, Pedro Garavito, Carolina Gil, Brian Hettler, Eduardo Londoño, Juanita Londoño, Jorge Hernán López, Ricardo Rey, Jerónimo Rodríguez Escobar, Alejo Sauna Mamatacán, Dignory Soto Londoño, José de los Santos Sauna, José Shibulata Zarabata, Carlos Vieira et Jacinto Zarabata.

Le processus a bénéficié du travail réalisé par le Conseil canadien des aires écologiques (CCAE) pour élaborer des orientations sur d'autres mesures de conservation efficaces par zone (MacKinnon et al., 2015), et a été complété par des travaux dirigés par BirdLife International et ses partenaires concernant la relation entre les Zones clés pour la biodiversité (ZCB), les aires protégées et les autres mesures de conservation efficaces par zone (BirdLife, 2017). Des ateliers ont été organisés au Kenya, en Colombie, aux Bermudes et en Afrique du Sud pour tester les versions antérieures des orientations. Un Numéro spécial de la revue PARKS (IUCN/WCPA, 2018) donne quelques exemples d'études de cas : voir https://parksjournal.com/list-of-papers/.

Pour plus d'informations sur le Groupe de travail, y compris tous les résultats et études de cas, veuillez consulter : www.iucn.org/ theme/protected-areas/wcpa/what-we-do/oecms.

Veuillez adresser vos questions ou commentaires à : oecm@wcpa.iucn.org 


\section{Glossaire}

\begin{abstract}
Aire marine d'importance écologique ou biologique : Les AlEB sont des zones océaniques spéciales dont le rôle est important pour soutenir, d'une manière ou d'une autre, le bon fonctionnement des océans et les nombreux services qu'ils fournissent. (https://www.cbd.int/ebsa/).
\end{abstract}

\begin{abstract}
Aire marine gérée localement : Une aire marine gérée localement (LMMA) est une zone d'eaux à proximité du littoral ainsi que ses ressources côtières et marines associées, et qui est en grande partie ou entièrement gérée au niveau local par les communautés côtières, les groupes propriétaires de terrains, les organisations partenaires, et/ou les représentants gouvernementaux collaboratifs qui résident ou sont basés dans les environs immédiats. (http://Immanetwork.org/).
\end{abstract}

Aire protégée : La CDB définit une aire protégée comme suit : "Toute zone géographiquement délimitée qui est désignée, ou réglementée, et gérée en vue d'atteindre des objectifs spécifiques de conservation » (Article 2 de la CDB). L'UICN propose une définition plus détaillée : "Un espace géographique clairement délimité, reconnu, consacré et géré, par tout moyen efficace, juridique ou autre, afin d'assurer à long terme la conservation de la nature ainsi que les services écosystémiques et les valeurs culturelles qui lui sont associés » (Dudley, 2008). La CDB et I'UICN reconnaissent que ces deux définitions sont équivalentes en pratique (Lopoukhine \& Dias, 2012) car dans les deux cas, ces aires ont vocation à apporter une conservation in situ.

\section{Aires conservées : Les Parties à la CDB et d'autres} organisations font de plus en plus référence aux « aires protégées et conservées » (voir par exemple la Décision 14/8 de la CDB et la Liste verte des aires protégées et conservées de l'UICN). Dans ce contexte, les " aires conservées » incluent des zones qui peuvent satisfaire aux critères des " autres mesures de conservation efficaces par zone ».

\section{AMCE candidate : Un espace géographiquement délimité} qui a été recensé en tant qu' «AMCE potentielle " par l'autorité de gouvernance, et que cette autorité a consenti à évaluer par rapport aux critères de la CDB.

AMCE potentielle : Un espace géographiquement délimité qui, après application de l'outil de présélection, a été identifié comme ayant des caractéristiques similaires à celles des AMCE, mais que l'autorité de gouvernance n'a pas encore validé en tant qu'« AMCE candidate ».

Approche écosystémique : L'approche écosystémique est une stratégie pour la gestion intégrée des terres, des eaux et des ressources vivantes, qui favorise la conservation et l'utilisation durable d'une manière équitable. L'application de l'approche écosystémique aidera à assurer l'équilibre entre les trois objectifs de la Convention. Cette approche repose sur l'application de méthodes scientifiques appropriées aux divers niveaux de l'organisation biologique, qui incluent les processus, les fonctions et les interactions essentiels entre les organismes et leur environnement. Elle reconnaît que les êtres humains, avec leur diversité culturelle, font partie intégrante des écosystèmes. (https://www.cbd.int/ecosystem/).

Autorité de gouvernance : L'institution, l'individu, les Populations autochtones ou le groupe communautaire, ou tout autre organe reconnu comme ayant autorité et responsabilité dans la prise de décisions et la gestion d'une zone.

Biodiversité : Variabilité des organismes vivants de toute origine y compris, entre autres, les écosystèmes terrestres, marins et autres écosystèmes aquatiques et les complexes écologiques dont ils font partie ; cela comprend la diversité au sein des espèces et entre espèces ainsi que celle des écosystèmes. (Article 2 de la CDB).

\section{Consentement préalable, donné librement et en} connaissance de cause : II s'agit d'un droit spécifique concernant les Populations autochtones et qui est reconnu dans la Déclaration des Nations Unies sur les droits des peuples autochtones. II leur permet de donner ou de refuser leur consentement au sujet de projets susceptibles de les affecter ou d'avoir une incidence sur leurs territoires. Une fois qu'ils ont donné leur consentement, ces populations peuvent le retirer à tout moment. En outre, ce « consentement préalable, donné librement et en connaissance de cause » leur permet de négocier les conditions selon lesquelles le projet sera conçu, mis en œuvre, suivi et évalué. Ceci est également intégré au droit universel à l'autodétermination. (United Nations, 2007).

Conservation in situ : La conservation des écosystèmes et des habitats naturels et le maintien et la reconstitution de populations viables d'espèces dans leur milieu naturel et, dans le cas des espèces domestiquées et cultivées, dans le milieu où se sont développés leurs caractères distinctifs. (Article 2 de la CDB).

Écosystème : Le complexe dynamique formé de communautés de plantes, d'animaux et de micro-organismes et de leur environnement non vivant qui par leur interaction, forment une unité fonctionnelle. (Article 2 de la CDB).

Géodiversité : L'aire de répartition naturelle (diversité) des caractéristiques géologiques (roches, fossiles, minéraux), géomorphologiques (relief, processus physiques) et des sols, ainsi que leurs assemblages, relations, propriétés et systèmes. (Gray, 2004).

Habitat : Le lieu ou type de site dans lequel un organisme ou une population existe à l'état naturel. (Article 2 de la CDB).

Organe de gestion : L'organisation ou entité responsable de la gestion continue d'un site. L'organe de gestion peut ou non être le même que l'autorité de gouvernance, l'organisation ou l'entité qui détient l'autorité légale ou coutumière et la responsabilité du site.

Populations autochtones et communautés locales : $\mathrm{Ce}$ rapport applique les termes « Populations autochtones » et « 
communautés locales " utilisés par la Convention sur la diversité biologique.

Utilisation durable : L'utilisation des éléments constitutifs de la diversité biologique d'une manière et à un rythme qui n'entraînent pas leur appauvrissement à long terme, et sauvegardent ainsi leur potentiel pour satisfaire les besoins et les aspirations des générations présentes et futures. (Article 2 de la CDB).

Valeurs culturelles et spirituelles : Celles-ci comprennent les valeurs récréatives, religieuses, esthétiques, historiques et sociales liées aux avantages tangibles et intangibles que la nature et les caractéristiques naturelles ont pour les personnes de cultures et de sociétés différentes, en mettant particulièrement l'accent sur celles qui contribuent aux résultats pour la conservation (par exemple, les pratiques de gestion traditionnelles envers lesquelles les espèces clés, la biodiversité ou des écosystèmes entiers sont devenus dépendants, ou bien le soutien de la société envers la conservation des paysages terrestres pour préserver leur qualité en termes de beauté ou d'expression artistique), ainsi que le patrimoine immatériel dont les pratiques culturelles et spirituelles. 


\section{Acronymes}

\begin{tabular}{|c|c|}
\hline AIEB & Aire marine d'importance écologique ou biologique \\
\hline AMCE & Autre mesure de conservation efficace par zone \\
\hline APAC & Aires et territoires du patrimoine autochtone et communautaire local \\
\hline CdP & Conférence des Parties à la CDB \\
\hline CMAP & Commission mondiale des aires protégées de l'UICN \\
\hline LMMA & Aire marine gérée localement (Locally Managed Marine Area) \\
\hline ODD & Objectifs de développement durable des Nations Unies \\
\hline ONU & Organisation des Nations Unies \\
\hline OSASTT & Organe subsidiaire chargé de fournir des avis scientifiques, techniques et technologiques de la CDB \\
\hline UICN & Union internationale pour la conservation de la nature et de ses ressources \\
\hline WCC & Congrès mondial de la nature de I'UICN (World Conservation Congress) \\
\hline WDPA & Base de données mondiale sur les aires protégées (World Database on Protected Areas) \\
\hline ZCB & Zone clé pour la biodiversité \\
\hline ZICO & Zone importante pour la conservation des oiseaux \\
\hline ZIP & Zone importante pour les plantes \\
\hline
\end{tabular}

\section{Liste des encadrés, tableaux et figures}

Encadré 1 : Recensement et établissement des autres mesures de conservation efficaces par zone 3

Encadré 2 : Gros plan sur l'espace géographique 5

Encadré 3 : Sites d'épaves historiques comme Flow, un exemple de conservation dérivée 6

Encadré 4 : Gros plan sur la biodiversité 7

Encadré 5 : Utilisation de l'outil de présélection : points clés à retenir 9

Encadré 6 : Vérification de la pertinence de l'Objectif 11 d'Aichi 10

Encadré 7 : Restauration écologique dans les AMCE 12

Tableau 1 : Principes de base pour la vérification des données à inclure dans les bases de données de Protected Planet 13

Figure 1 : La relation entre les AMCE et les aires protégées 4 


\section{Introduction}

\section{Contexte}

Le Plan stratégique pour la biodiversité 2011-2020 est un cadre pour la mise en œuvre efficace de la Convention sur la diversité biologique (CDB) par une approche stratégique, comprenant une vision partagée, une mission, des buts stratégiques et des objectifs ("Objectifs d'Aichi pour la biodiversité »), ce qui a inspiré des actions de grande envergure par tous les pays et parties prenantes. L'Objectif 11 d'Aichi relève du But stratégique C, lequel vise à améliorer l'état de la diversité biologique en sauvegardant les écosystèmes, les espèces et la diversité génétique. L'Objectif 11 indique ceci :

D'ici à 2020, au moins $17 \%$ des zones terrestres et d'eaux intérieures et $10 \%$ des zones marines et côtières, y compris les zones qui sont particulièrement importantes pour la diversité biologique et les services fournis par les écosystèmes, sont conservées au moyen de réseaux écologiquement représentatifs et bien reliés d'aires

protégées gérées efficacement et équitablement et d'autres mesures de conservation efficaces par zone, et intégrées dans l'ensemble du paysage terrestre et marin [emphase ajoutée].

Les aires protégées constituent le fondement des stratégies nationales de conservation de la biodiversité et de la réalisation de l'Objectif 11 (Watson et al., 2014). L'UICN a fourni des orientations sur la définition, les catégories de gestion et les modes de gouvernance des aires protégées (Dudley 2008 ; Borrini-Feyerabend et al., 2014). Les Parties à la Convention sur la diversité biologique (CDB) ont inclus les « autres mesures de conservation efficaces par zone » (AMCE) dans l'Objectif 11 car certaines zones situées hors des réseaux d'aires protégées reconnues produisent aussi des résultats efficaces pour la conservation in situ de la biodiversité. Ces zones peuvent inclure des territoires et aires conservées faisant l'objet d'une gouvernance appartenant à l'un de ces quatre modes : gouvernance par les gouvernements, par des acteurs privés, par les Populations autochtones et communautés locales, ou bien gouvernance partagée. Quelles que soient les réalisations dans le cadre de l'Objectif 11 d'ici 2020, il est probable que les aires protégées et les AMCE feront partie des objectifs pour l'après 2020 en vue de conserver la biodiversité in situ, et que les AMCE deviendront un outil utilisé plus couramment dans les stratégies de conservation.

Depuis 2010, les Parties à la CDB ont fait des progrès importants dans l'expansion des réseaux d'aires protégées, y compris la déclaration de nombreuses aires marines protégées très vastes (UNEP-WCMC \& IUCN, 2016, 2018). Les progrès ont été plus lents en matière de définition, de recensement, de reconnaissance et d'établissement de rapports concernant les AMCE (Jonas et al., 2014). L'Union internationale pour la conservation de la nature (UICN) et la Commission mondiale des aires protégées (CMAP) de l'UICN ont été invitées par les Parties à la CDB à " continuer à élaborer des orientations techniques pour réaliser l'ensemble de l'Objectif 11 d'Aichi relatif à la diversité biologique » (Décision
XI/24 de la CDB). En 2015, la CMAP a mis en place un Groupe de travail chargé d'élaborer des orientations techniques sur les AMCE. La version préliminaire des Lignes directrices préparées par le Groupe de travail a été transmise au Secrétariat de la CDB et partagée avec les Parties à la CDB, puis discutée lors de deux ateliers organisés par la CDB (février 2018) et présentée lors d'événements en marge des réunions de l'Organe subsidiaire chargé de fournir des avis scientifiques, techniques et technologiques de la CDB (2016-2018). De plus amples informations sur le processus d'élaboration des lignes directrices sont disponibles dans un Numéro spécial de la revue PARKS sur les AMCE (Jonas et al., 2018).

Lors de la $14^{\mathrm{e}}$ Conférence des Parties à la CDB (CdP 14) en novembre 2018, les Parties ont adopté une décision sur les « Aires protégées et autres mesures de conservation efficaces par zone » (CBD/COP/DEC/14/8, https://www.cbd.int/doc/ decisions/cop-14/cop-14-dec-08-fr.pdf). Cette décision a adopté une définition des « autres mesures de conservation efficaces par zone » et des critères pour leur recensement et leur gestion.

La Décision 14/8 (paragraphe 9) a de plus invité l'UICN et d'autres organismes spécialisés à continuer d'aider les Parties à identifier des AMCE et à appliquer l'avis scientifique et technique pour leur recensement.

\section{Élaboration des lignes directrices}

Les présentes lignes directrices ont été élaborées par le Groupe de travail de la CMAP-UICN sur les autres mesures de conservation efficaces par zone, en réponse à la CdP 14 qui demandait d'aider les Parties à rendre la Décision 14/8 opérationnelle. Elles ont été conçues pour une application à diverses échelles, par exemple pour comprendre si une zone individuelle est une AMCE, ou pour établir des rapports statistiques sur les AMCE au niveau national et mondial afin d'évaluer les progrès enregistrés pour atteindre les objectifs de conservation. La préparation de ces lignes directrices a bénéficié du travail réalisé par le Conseil canadien des aires écologiques (CCAE) pour élaborer des orientations sur d'autres mesures de conservation efficaces par zone (MacKinnon et al., 2015), et a été complétée par des travaux dirigés par BirdLife International et ses partenaires concernant la relation entre les Zones clés pour la biodiversité (ZCB) (IUCN, 2016), les aires protégées et les AMCE (Donald et al., 2019).

Ces lignes directrices s'adressent principalement aux Parties à la CDB, aux agences gouvernementales, aux organismes des Nations Unies (ONU), aux organisations non gouvernementales (ONG), aux organismes privés, aux organisations de Populations autochtones, aux communautés locales et aux autres organisations, organismes et individus intéressés pour comprendre, appliquer et suivre les progrès réalisés pour atteindre l'Objectif 11 d'Aichi et d'autres objectifs 
de conservation. Les AMCE contribueront aussi, directement et indirectement, à la réalisation de plusieurs Objectifs de développement durable (ODD) des Nations Unies. La mise en œuvre permettra en outre d'éclairer le processus de la CDB pour l'élaboration du Cadre mondial de la biodiversité pour l'après-2020 et la réalisation des ODD, en particulier dans le contexte des approches émergentes en matière de conservation du paysage terrestre et marin (CBD, 2019). Ces lignes directrices s'appliquent à l'ensemble des milieux terrestres, marins et d'eau douce. En tant que telles, elles devraient éclairer d'autres processus, y compris les discussions sur un traité juridiquement contraignant pour la mise en œuvre d'aires marines protégées en haute mer.

\section{Contenu des lignes directrices}

Ces lignes directrices fournissent des informations sur la façon d'appliquer la définition des AMCE au niveau de conservation international, national, infranational ou local, et d'établir des rapports sur les AMCE à l'attention de la Base de données mondiale sur les aires protégées (WDPA) et de la CDB.

Elles expliquent la définition du terme " autres mesures de conservation efficaces par zone ", ainsi que les approches et outils recommandés pour le recensement, la reconnaissance, le suivi et l'établissement de rapports. D'autres sections traitent de la relation avec d'autres Objectifs d'Aichi pour la biodiversité, des différences entre les aires protégées et les AMCE, et de la façon de rendre compte à la Base de données mondiale sur les aires protégées (WDPA). Tenant compte de la Décision 14/8, ces lignes directrices indiquent comment les AMCE sont appliquées dans le cadre de principes existants énoncés par la CDB, I'UICN et les partenaires, en ce qui concerne la conservation de la biodiversité, les droits de l'homme et le développement durable.

En aboutissant à la conservation in situ efficace de la biodiversité, les AMCE peuvent contribuer à maintenir les valeurs existantes de la biodiversité et à améliorer les résultats pour la conservation de la biodiversité ; par exemple, en préservant les écosystèmes, habitats et corridors fauniques importants, en soutenant la récupération des espèces menacées, en maintenant les fonctions des écosystèmes et en sécurisant les services écosystémiques, en améliorant la résilience face aux menaces, et enfin en préservant et en mettant en relation les vestiges d'écosystèmes fragmentés dans les paysages terrestres dégradés. Les AMCE peuvent aussi contribuer à des réseaux de conservation écologiquement représentatifs et bien reliés, et intégrés dans l'ensemble du paysage terrestre et marin.

Le recensement des AMCE et la reconnaissance de leurs structures de gouvernance et de gestion offrent l'occasion de faire participer et soutenir un ensemble de nouveaux partenaires dans les efforts de conservation à l'échelle mondiale. Dans certains contextes, cela permet un dialogue et favorise la réconciliation grâce à la reconnaissance appropriée des « aires protégées et conservées autochtones » (Indigenous Circle of Experts, 2018). La reconnaissance en tant qu'AMCE peut également fournir des incitations supplémentaires pour la conservation et la gestion durable des zones d'importance pour la biodiversité en dehors des aires protégées, comme les Zones clés pour la biodiversité (ZCB), les Zones importantes pour les plantes (ZIP), les Zones importantes pour la conservation des oiseaux (ZICO), les Aires importantes pour les mammifères marins (IMMA) et les Aires marines d'importance écologique ou biologique (AlEB), en soulignant que ces zones doivent correspondre à la définition d'une AMCE pour être reconnues en tant que telles. L'application de ces lignes directrices peut aussi contribuer à l'amélioration de la gouvernance ou de la gestion des AMCE candidates.

2 | Reconnaissance et signalement des autres mesures de conservation efficaces par zone 


\section{Définition et caractéristiques}

Cette section précise la définition d'une " autre mesure de conservation efficace par zone " et fournit des orientations sur chaque élément de la définition.

\subsection{Définition d'une " autre mesure de conservation efficace par zone »}

Une « autre mesure de conservation efficace par zone » (AMCE) est définie comme suit par la CDB dans la Décision 14/8 :

Une zone géographiquement délimitée, autre qu'une aire protégée, qui est réglementée et gérée de façon à obtenir des résultats positifs et durables à long terme pour la conservation in situ de la diversité biologique, y compris des fonctions et services écosystémiques connexes et, le cas échéant, des valeurs culturelles, spirituelles, socioéconomiques et d'autres valeurs pertinentes localement. (CBD, 2018).

Cette définition d'une AMCE complète la définition de I'UICN du terme « aire protégée » (Dudley, 2008). L'UICN définit une aire protégée comme suit :

Un espace géographique clairement délimité, reconnu, consacré et géré, par tout moyen efficace, juridique ou autre, afin d'assurer à long terme la conservation de la nature ainsi que les services écosystémiques et les valeurs culturelles qui lui sont associés.

La distinction entre les deux réside dans le fait qu'une aire protégée a un objectif de conservation primaire, alors qu'une " autre mesure de conservation efficace par zone " aboutit à la conservation in situ efficace de la biodiversité, indépendamment de ses objectifs.

\section{Encadré 1}

\section{Recensement et établissement des autres mesures de conservation efficaces par zone}

Les aires protégées et les AMCE devraient toutes les deux aboutir à la conservation in situ efficace de la biodiversité à long terme. Toutefois, alors que les aires protégées ont la conservation de la nature comme objectif de gestion primaire, les AMCE n'ont pas nécessairement la conservation de la nature comme objectif.

\section{Types d'approches aboutissant à la conservation efficace} dans les AMCE

1. La " conservation primaire »: désigne les zones qui peuvent répondre à tous les éléments de la définition de I'UICN d'une aire protégée, mais qui ne sont pas officiellement désignées comme telles parce que l'autorité de gouvernance ne veut pas que la zone soit reconnue ou signalée en tant qu'aire protégée. Par exemple, dans certains cas, les Populations autochtones et communautés locales peuvent ne pas souhaiter que les zones à forte valeur de biodiversité dont ils assurent la gouvernance soient désignées en tant qu'aires protégées, ou enregistrées dans des bases de données gouvernementales sur les aires protégées. En supposant qu'une zone réponde aux critères des AMCE, l'autorité de gouvernance a le droit de refuser qu'une zone soit reconnue en tant qu'AMCE, ou il peut donner son consentement.

2. La " conservation secondaire " : celle-ci est réalisée par la gestion active d'une zone où les résultats pour la biodiversité sont un objectif de gestion secondaire. Par exemple, une gestion et des politiques durables pour la protection des bassins versants peuvent conduire à une protection efficace de la biodiversité dans les bassins versants, même si les zones peuvent être gérées principalement pour des objectifs autres que la conservation. Les sites gérés pour fournir une connectivité écologique entre les aires protégées ou d'autres zones à forte biodiversité, contribuant ainsi à leur viabilité, peuvent également être admissibles en tant qu'AMCE.

3. La « conservation dérivée " : désigne les zones qui aboutissent à la conservation in situ en tant que « produit dérivé " des activités de gestion, même si la conservation de la biodiversité n'est pas un objectif de gestion. Par exemple, Scapa Flow dans les Orcades protège les épaves et le cimetière militaire sous-marin. Cette protection a conduit à la conservation dérivée d'une biodiversité importante (voir Encadré 3).

\section{Zones non reconnues et non signalées correspondant à la} définition d'une aire protégée

L'UICN recommande que les zones répondant à tous les éléments de la définition de l'UICN d'une aire protégée, et qui sont reconnues comme telles par l'autorité de gouvernance, soient considérées en tant qu'aires protégées et non en tant qu'AMCE (voir Figure 1 et Section 4). Par exemple, certaines aires protégées privées ne sont pas signalées en tant qu'aires protégées par les gouvernements nationaux, même si elles peuvent correspondre aux critères de I'UICN.

\section{Autres espaces naturels intacts}

Tous les cas ci-dessus doivent être distingués des autres espaces naturels intacts qui ne sont pas soumis à une forme délibérée de gestion de la conservation, mais qui abritent toutefois une biodiversité intacte actuellement, par exemple en raison de leur éloignement ou de situations de conflit. Ces espaces ne sont considérés ni en tant qu'AMCE ni en tant qu'aires protégées, car ces sites peuvent présenter une faible sécurité à long terme si les conditions changent, ou bien s'ils sont exposés à des activités dommageables pour l'environnement. 


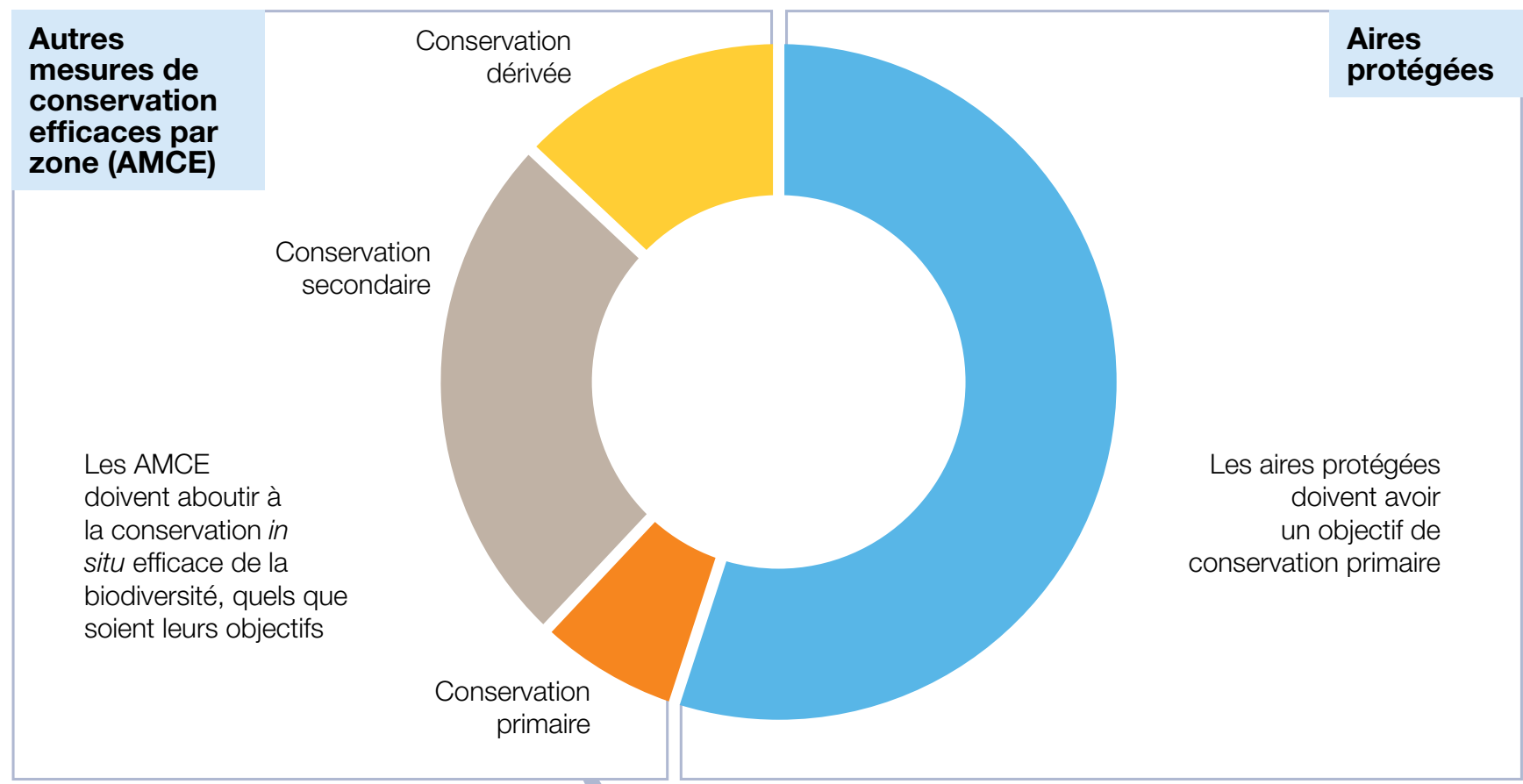

Un site ayant un objectif de conservation primaire passerait d'AMCE à Aire protégée s'il est reconnu en tant qu'aire protégée par l'autorité de gouvernance correspondante

Figure 1. La relation entre les AMCE et les aires protégées (remarque : les segments sont proposés

à des fins d'illustration uniquement et leur taille ne correspond pas aux données réelles).

Il existe plusieurs raisons pour lesquelles les zones produisant d'importants résultats pour la conservation in situ peuvent ne pas être reconnues et signalées en tant qu'aires protégées (BorriniFeyerabend \& Hill, 2015) alors que ces zones peuvent être reconnues par le biais de processus de validation appropriés en tant qu'AMCE (voir Encadré 1). Même si les aires protégées et les AMCE contribuent à la réalisation de l'Objectif 11 d'Aichi, elles présentent un certain nombre d'autres différences importantes.

La relation entre les AMCE et les aires protégées est présentée à la Figure 1

\section{2 Éléments de la définition}

Les paragraphes suivants décrivent chaque élément de la définition générale d'une « autre mesure de conservation efficace par zone » :

Une zone géographiquement délimitée, autre qu'une aire protégée, qui est réglementée et gérée de façon à obtenir des résultats positifs et durables à long terme pour la conservation in situ de la diversité biologique, y compris des fonctions et services écosystémiques connexes et, le cas échéant, des valeurs culturelles, spirituelles, socioéconomiques et d'autres valeurs pertinentes localement.

Les éléments sont présentés selon les quatre critères, conformément à la Décision 14/8.

\section{Critère A : L'aire n'est pas reconnue comme une aire protégée à l'heure actuelle}

\section{a. « autre qu'une aire protégée "}

Les AMCE peuvent contribuer de leur propre droit à des objectifs de conservation terrestre, marine et d'eau douce par zone. Cela signifie que les zones déjà désignées en tant qu'aires protégées ou se trouvant au sein d'aires protégées ne doivent pas être reconnues ou signalées en tant qu'AMCE. Même si les aires protégées et les AMCE peuvent s'exclure mutuellement, les aires protégées et les AMCE ont toutes les deux de la valeur pour la conservation de la biodiversité. Certaines AMCE peuvent devenir reconnues en tant qu'aires protégées si, par exemple, la conservation de la nature devient l'objectif de gestion primaire, ou lorsque la zone correspond déjà à la définition d'une aire protégée et que l'autorité de gouvernance demande maintenant sa reconnaissance.

\section{Critère B : L'aire fait l'objet d'une gouvernance et d'une gestion}

\section{b. “ zone géographiquement délimitée "}

Le terme "zone géographiquement délimitée » implique une zone déterminée dans l'espace avec des frontières convenues et délimitées, et pouvant inclure des terres, des eaux intérieures, des aires marines et côtières, ou la conjugaison de celles-ci. Dans des cas exceptionnels, les frontières peuvent être délimitées par des caractéristiques physiques qui évoluent au fil du temps, telles que les rives des rivières, la laisse de haute mer ou l'étendue de la glace de mer (voir Encadré 2). 
Encadré 2

\section{Gros plan sur l'espace géographique}

L'espace géographique a trois dimensions. Ainsi, pour conserver efficacement in situ l'ensemble de la biodiversité d'une zone, tout régime de gouvernance ou de gestion s'appliquant à une zone à deux dimensions doit également tenir compte de la troisième dimension (verticale). Les désignations des aires protégées ou des AMCE auront souvent des limites concernant la troisième dimension (par exemple, en s'appliquant uniquement à une certaine profondeur dans le sous-sol ou en dessous de la surface de l'eau, ou bien en ayant une limite d'altitude pour permettre le passage des avions commerciaux). Cela est devenu particulièrement controversé dans les aires marines protégées, où le zonage vertical à des fins commerciales nuit aux résultats pour la conservation, perturbe la connectivité écologique et crée des difficultés en matière de suivi et d'application. Pour les aires protégées comme pour les AMCE, les dimensions en hauteur et en profondeur doivent être compatibles avec une gestion efficace de la conservation, afin de protéger l'intégralité de la biodiversité autochtone. En conséquence, I'UICN a une forte présomption contre le zonage vertical des AMCE.
Même si la taille des AMCE peut varier, elle devrait être de taille suffisante pour aboutir à la conservation in situ de la biodiversité à long terme, y compris tous les écosystèmes, habitats et communautés d'espèces pour lesquels le site est important. La notion de « taille suffisante " est très contextuelle et dépend des exigences écologiques pour la persistance des espèces et écosystèmes concernés.

\section{c. « réglementée »}

Le terme "réglementée » implique que l'aire est sous l'autorité d'une entité spécifiée ou d'un ensemble d'entités convenu. Les AMCE peuvent être réglementées par les mêmes modes de gouvernance que les aires protégées, à savoir :

1. La gouvernance par les gouvernements (à différents niveaux) ;

2. La gouvernance par des particuliers, des organisations ou des entreprises ;

3. La gouvernance par les Populations autochtones et/ou communautés locales ; et

4. La gouvernance partagée (c.-à-d. gouvernance par divers détenteurs de droits et parties prenantes travaillant ensemble) (Dudley, 2008 ; Borrini-Feyerabend et al., 2014).

As with protected areas, the governance of OECMs should be equitable and reflect human rights principles recognised in international and regional human rights instruments and in national legislation, including relating to gender equity and indigenous peoples. Governance mechanisms should be effective in maintaining biodiversity. Any recognition or reporting of OECMs governed by indigenous peoples and/or local communities should be based on self-identification and requires the free, prior and informed consent of those traditional governance authority(ies) (United Nations, 2007).

\section{d. "gérée »}

Le terme « gérée " précise que l'aire est gérée de façon à obtenir des résultats positifs et durables à long terme pour la conservation de la diversité biologique. Les autorités compétentes, les détenteurs de droits et les parties prenantes devraient être connus et participer à la gestion.

Contrairement aux aires protégées, les AMCE n'ont pas besoin d'un objectif de conservation primaire, mais il doit y avoir un lien de causalité direct entre, d'une part, l'objectif global et la gestion de la zone, et, d'autre part, la conservation in situ de la biodiversité à long terme. Le terme " gérée » peut inclure une décision délibérée de laisser la zone intacte - voir l'exemple des sites d'épaves historiques (Encadré 3).

\section{La gestion des AMCE devrait être conforme à l'approche} par écosystème et être adaptée pour obtenir les résultats de conservation escomptés à long terme pour la diversité biologique, avec la capacité de faire face aux nouvelles menaces (https:// www.cbd.int/ecosystem/). En conséquence, la gestion des AMCE devrait inclure des " moyens efficaces " de contrôle des activités qui pourraient avoir des effets sur la biodiversité, que ce soit par des mesures juridiques ou d'autres moyens efficaces (comme les lois coutumières ou des accords contraignants avec les propriétaires fonciers). Le cas échéant et dans la mesure du possible, la gestion devrait être intégrée dans toutes les AMCE et intégrée aux zones environnantes.

Une zone dépourvue de régime de gestion n'est pas une AMCE, même si sa biodiversité peut rester intacte. Par exemple, les zones non gérées en haute mer, les zones de conflit militaire et les autres zones actuellement dans un état naturel ou quasi naturel ne devraient pas être considérées en tant qu'AMCE en l'absence d'un régime de gestion qui assure une conservation in situ efficace et durable de la diversité biologique. Les régimes de gestion peuvent inclure des décisions délibérées de laisser la zone intacte.

\section{Critère $C$ : Apporte une contribution durable et efficace à la conservation in situ de la diversité biologique}

\section{e. " résultats positifs " pour la conservation de la diversité biologique (le terme " efficaces " est employé dans les critères de la Décision $14 / 8$ de la CDB)}

Les AMCE devraient être efficaces pour obtenir des résultats positifs durables pour la conservation in situ de la diversité biologique. Plus précisément, il devrait y avoir un lien clair entre la gestion et les résultats pour la biodiversité, avec des mécanismes en place pour répondre aux menaces existantes ou anticipées (voir Mathur et al., 2017 pour des orientations sur l'identification et la gestion des menaces).

Les activités industrielles et le développement d'infrastructures portant préjudice à l'environnement ne devraient pas se produire dans les AMCE. Ceci est conforme à la Recommandation 102 de I'UICN (WCC-2016-Rec-102-FR), adoptée lors Congrès mondial de la nature 2016 à Hawaï. Cette recommandation appelle les gouvernements et autorités compétentes « à adopter et appliquer des politiques qui limitent les activités industrielles et le développement d'infrastructures portant préjudice à l'environnement pouvant avoir des effets négatifs sur (...) toute zone d'importance particulière pour la biodiversité et les services écosystémiques que les gouvernements ont jugés essentiels pour atteindre les Objectifs d'Aichi pour la biodiversité ». Les activités 
Encadré 8

\section{Sites d'épaves historiques comme Flow, un exemple de conservation dérivée}

La protection stricte des sites d'épaves historiques à des fins culturelles et historiques est une caractéristique commune dans de nombreux bassins océaniques à travers le monde. Cette finalité aboutit par la même occasion à la protection des habitats, espèces et écosystèmes marins associés. II y a de nombreux exemples dans les Caraïbes et dans l'océan Pacifique, témoignant des conflits historiques à travers les âges. Au Royaume-Uni, Scapa Flow est peut-être l'exemple le plus connu d'une telle " conservation dérivée ".

Scapa Flow est un port naturel au large de la partie continentale des Orcades, dans le nord de l'Écosse. La zone est sous la juridiction de l'administration portuaire des Orcades, dont les objectifs de gestion pour cette zone sont la gestion sécuritaire du port tout en préservant le patrimoine culturel du site. La zone est connue pour les épaves de navires de guerre allemands sabordés dans le port pendant la Première Guerre mondiale et celle du Royal Oak, le vaisseau amiral de la Royal Navy coulé par un U-Boat allemand pendant la Seconde Guerre mondiale, et est un cimetière militaire sous-marin désigné.

Scapa Flow couvre une superficie de $324,5 \mathrm{~km}^{2}$, avec un volume d'eau d'environ 1 milliard de mètres cubes. La protection stricte accordée à ces épaves historiques offre par la même occasion un haut niveau de protection à l'écosystème benthique, comme en témoignent les bancs de maërl, les bancs de limes bâillantes, les récifs de modioles (grandes moules) et les grandes nacres, ces espèces y étant florissantes alors qu'elles sont très rares dans le reste de l'Écosse. Bien que la zone ne soit pas gérée avec un objectif spécifique de conservation de la nature, la protection de la biodiversité du site est assurée grâce à la conservation dérivée. En février 2019, la Première ministre écossaise, Nicola Sturgeon, a annoncé une consultation sur deux nouvelles aires marines protégées (AMP) « historiques » en Écosse, l'une étant Scapa Flow. En cas d'approbation, cela démontrerait l'étroite relation entre les AMCE et les aires protégées.

industrielles portant préjudice à l'environnement comprennent, par exemple, la pêche et la sylviculture industrielles, l'exploitation minière, gazière et pétrolière, l'agriculture industrielle, et les infrastructures portant préjudice à l'environnement, comme les barrages, les routes, les oléoducs et gazoducs. Ces menaces devraient être évitées. Cela s'applique aux activités portant préjudice à l'environnement non seulement au sein des AMCE mais aussi en dehors de la zone avec une incidence sur les AMCE.

\section{f. “ durables à long terme »}

La gouvernance et la gestion des AMCE devraient être durables et aboutir à la conservation in situ efficace de la biodiversité à long terme. Les stratégies de gestion temporaires ou à court terme ne constituent pas une AMCE. Par exemple, une fermeture de la pêche commerciale maintenue uniquement jusqu'à la reconstitution d'une zone de surpêche n'est pas une AMCE. Selon les orientations de l'UICN, les facteurs attendus pour réglementer et gérer une AMCE devraient être continus et à long terme.
Des résultats efficaces pour la conservation peuvent découler d'une protection stricte ou de certaines formes de gestion durable, conformes aux définitions des termes " conservation in situ » et « diversité biologique " prévues par la CDB. Toutefois, la plupart des aires gérées pour la production industrielle, même si elles offrent des avantages pour la diversité biologique, ne devraient pas être considérées en tant qu'AMCE. Par exemple, la pêche commerciale et l'exploitation commerciale des forêts dont la gestion est durable devraient être signalées dans le cadre des Objectifs 6 et 7 d'Aichi, respectivement, ou d'autres objectifs appropriés.

Par contre, les sites ayant diverses approches en termes de gestion, y compris les dispositions saisonnières (par exemple, les sites gérés pour les espèces d'oiseaux migrateurs), pourraient être admissibles en tant qu'AMCE si les mesures saisonnières font partie d'un régime global de gestion à long terme qui aboutit, tout au long de l'année, à la conservation in situ de la biodiversité pour laquelle le site est important. Dans certains cas, les instruments réglementaires à court terme, renouvelés continuellement, peuvent fournir des mesures de facto à long terme.

La gestion des AMCE devrait s'effectuer dans le respect d'une approche par écosystème et de précaution, avec la capacité de s'adapter pour obtenir des résultats pour la diversité biologique à long terme et gérer de nouvelles menaces potentielles. Des mesures pratiques devraient être mises en place pour le suivi et l'établissement de rapports concernant l'efficacité des AMCE (voir Section 4).

\section{g. " conservation in situ de la diversité biologique »}

La CDB définit la conservation in situ, en ce qui concerne la biodiversité, comme suit :

La conservation des écosystèmes et des habitats naturels et le maintien et la reconstitution de populations viables d'espèces dans leur milieu naturel et, dans le cas des espèces domestiquées et cultivées, dans le milieu où se sont développés leurs caractères distinctifs. (Article 2 de la $C D B$ ).

Les autres mesures de conservation efficaces par zone devraient aboutir à des résultats pour la diversité biologique qui sont d'une importance comparable aux résultats obtenus par les aires protégées et qui les complètent. Cela inclut leur contribution à la représentativité écologique, la couverture des zones importantes pour la biodiversité et les fonctions et services écosystémiques connexes, la connectivité et l'intégration dans les paysages terrestres et marins plus vastes, ainsi que l'efficacité de la gestion et des exigences en matière d'équité.

\section{Les AMCE devraient aboutir à la conservation de la nature}

dans son ensemble, et pas seulement de certains éléments de la biodiversité. Les définitions de la CDB concernant la " diversité biologique " et la « conservation in situ » reconnaissent clairement qu'une seule espèce ne peut exister in situ qu'au sein d'un réseau interconnecté avec d'autres espèces et l'environnement abiotique. Par conséquent, il ne faudrait pas que les mesures de conservation visant des espèces individuelles ou des sousensembles de biodiversité compromettent l'écosystème au sens large. Reconnaissant le lien entre la diversité biologique et géologique, la " géodiversité » peut également être digne d'intérêt en matière de gestion des AMCE (Zarnetske et al., 2019). 


\section{Gros plan sur la biodiversité}

Les AMCE devraient protéger efficacement un ou plusieurs des éléments suivants de la biodiversité autochtone :

- Les espèces et habitats rares, menacés ou en danger, ainsi que les écosystèmes qui les soutiennent, y compris les espèces et les sites identifiés sur la Liste rouge des espèces menacées de l'UICN, la Liste rouge des écosystèmes, ou les équivalents nationaux.

- Les écosystèmes naturels représentatifs.

- Les zones à haut niveau d'intégrité écologique, caractérisées par la présence de l'ensemble des espèces autochtones et les processus écologiques à l'appui. Ces zones seront intactes ou restaurées dans le cadre du régime de gestion proposé.

- Les écosystèmes et espèces dont l'aire de répartition est restreinte, dans les milieux naturels.

- Les agrégats importants d'espèces, y compris en période de migration ou de frai.

- Les écosystèmes particulièrement importants pour les différents stades de vie des espèces, leur alimentation, leur repos, leur mue et leur reproduction.

- Les aires importantes pour la connectivité écologique ou pour compléter un réseau de conservation dans un paysage terrestre ou marin.
- Les zones fournissant des services écosystémiques critiques, comme l'eau propre et le stockage du carbone, en plus de la conservation in situ de la biodiversité.

- Les espèces et habitats importants pour les utilisations humaines traditionnelles, comme les plantes médicinales autochtones, en plus de la conservation in situ de la biodiversité.

Dans ce contexte, une ferme gérée de manière intensive avec une petite proportion de plantes et d'oiseaux autochtones d'origine ne sera probablement pas une AMCE. Inversement, une zone de prairies autochtones, riche en plantes indigènes, et ayant des populations saines d'une grande variété d'oiseaux et de mammifères autochtones, pourrait bien être une AMCE si un régime de gestion et de gouvernance de faible intensité assure ces résultats à long terme. Tout comme pour les aires protégées, il peut $y$ avoir des cas où une AMCE est particulièrement importante pour protéger une espèce menacée spécifique tout en protégeant l'écosystème tout entier.

Au fur et à mesure que le changement climatique altère les écosystèmes, la compréhension de ce qui est naturel et efficace dans un endroit donné peut aussi changer. II faudra peut-être reconnaître et gérer les AMCE en tenant compte de l'adaptation au changement climatique (Gross et al., 2016).

\section{h. « diversité biologique »}

Compte tenu du lien explicite entre les AMCE et les résultats pour la conservation de la biodiversité, il est clair que les AMCE doivent aboutir à la conservation in situ efficace et durable de la diversité biologique. Même si les approches pour recenser les éléments importants de la biodiversité dans ces zones varient selon les circonstances nationales, infranationales et locales, il existe maintenant des orientations mondiales pour identifier les Zones clés pour la biodiversité (IUCN, 2016) et décrire des zones, telles que les sites Ramsar et les Aires marines d'importance écologique ou biologique (Dunstan, 2016). La biodiversité conservée par une AMCE peut être présente dans des zones à l'intérieur ou au-delà de la juridiction nationale.

La reconnaissance d'une AMCE devrait inclure le recensement des différentes caractéristiques de la diversité biologique qui expliquent l'importance du site, et être basée sur les meilleures connaissances disponibles (voir Encadré 4). Ces valeurs clés de la biodiversité, ainsi que les valeurs de conservation plus larges des AMCE, devraient être décrites et suivies au fil du temps.

\section{Critère $D$ : Fonctions et services écosystémiques connexes, et valeurs culturelles, spirituelles, socioéconomiques et autres valeurs pertinentes localement}

\section{i. " fonctions et services écosystémiques "}

Des écosystèmes sains et en fonctionnement procurent un ensemble de services. Les fonctions écosystémiques font partie intégrante de la biodiversité et sont définies comme étant les processus biologiques, géochimiques et physiques qui ont lieu ou se produisent au sein d'un écosystème. Les services écosystémiques comprennent des services d'approvisionnement (nourriture, eau, etc.), des services de régulation (en cas d'inondation, de sécheresse, de dégradation des sols, de maladies, etc.) et des services de soutien (formation des sols, recyclage des nutriments, etc.). La protection de ces fonctions et services écosystémiques peut être une justification souvent appliquée pour la reconnaissance des AMCE. Toutefois, il ne faudrait pas que la gestion visant à améliorer un service écosystémique particulier ait des effets négatifs sur les valeurs globales du site en termes de conservation de la biodiversité.

\section{j.« des valeurs culturelles, spirituelles, socioéconomiques et d'autres valeurs pertinentes localement "}

Les AMCE incluent les zones où la protection des espèces et habitats clés ainsi que la gestion de la biodiversité peuvent être réalisées dans le cadre de pratiques et de valeurs culturelles, spirituelles, socioéconomiques et d'autres valeurs pertinentes localement. Dans de tels cas, il sera essentiel d'assurer la reconnaissance et la protection des liens entre la diversité biologique et culturelle et les pratiques de gouvernance et de gestion associées qui conduisent à des résultats positifs pour la biodiversité, comme l'usage coutumier des ressources biologiques selon une utilisation durable (Article 10(c) de la $\mathrm{CDB}$ ). Inversement, la gestion des valeurs culturelles, spirituelles, socioéconomiques ou d'autres valeurs pertinentes localement au sein d'une AMCE ne devrait pas avoir d'incidence négative sur les valeurs de conservation de la biodiversité. 


\section{Recensement des autres mesures de conservation efficaces par zone en pratique}

La conservation in situ de la biodiversité est « essentielle " pour endiguer l'appauvrissement de la diversité biologique (CBD, 1992). Les aires protégées et les AMCE sont les principaux moyens d'aboutir à la conservation in situ dans le cadre de l'Objectif 11 d'Aichi, et elles resteront des caractéristiques importantes pour les objectifs de la CDB après 2020. Tous les efforts de conservation de la biodiversité sont précieux, mais seules les mesures par zone contribuant directement à la conservation in situ à long terme devraient être prises en compte pour l'établissement de rapports au titre des objectifs de conservation in situ, comme l'Objectif 11 d'Aichi. D'autres efforts de conservation, y compris des approches par zone visant une utilisation durable, seront signalés de manière plus adaptée par rapport à d'autres types d'objectifs, tels que l'Objectif 6 d'Aichi (pêche durable) et l'Objectif 7 (sylviculture et agriculture durables), ou des objectifs analogues pour l'après 2020 (voir, par exemple, Laffoley et al., 2017 ou l'Annexe I). Le recensement des AMCE et l'établissement de rapports à leur sujet contribueront à tout objectif de la CDB en matière de conservation in situ, y compris l'Objectif 11 d'Aichi et les objectifs de conservation in situ adoptés après 2020.

Pour soutenir les processus décisionnels, la CMAP a élaboré un outil simple de présélection en quatre étapes, en lien direct avec la définition et l'explication des termes de la Section 2. Toute aire étudiée à des fins de reconnaissance en tant qu'AMCE doit d'abord être examinée pour déterminer son admissibilité à ces critères par, ou avec le consentement de, l'autorité de gouvernance.

\subsection{Présentation de l'outil de présélection}

L'outil de présélection (voir Encadré 5) applique quatre tests pour déterminer si une zone est admissible en tant qu'AMCE candidate.

- Test 1. S'assurer que la zone n'est pas déjà reconnue et/ou enregistrée en tant qu'aire protégée.

- Test 2. S'assurer que la zone présente les caractéristiques essentielles définies pour être une AMCE.

- Test 3. S'assurer que le résultat pour la conservation perdurera à long terme.

- Test 4. S'assurer qu'un objectif de conservation in situ par zone (par exemple, l'Objectif 11 d'Aichi), par opposition à un objectif d'utilisation durable, est la bonne priorité pour l'établissement d'un rapport.

Les éléments constituant chaque test sont détaillés à la Section 3.2. Une zone doit réussir les quatre tests de présélection pour être considérée en tant qu'AMCE candidate.
Encadré 5

\section{Utilisation de l'outil de présélection : points clés à retenir}

II y a sept points importants à prendre en compte et à traiter lors de l'application de l'outil de présélection :

1. Dans les cas où une partie autre que l'autorité de gouvernance gère le processus - y compris les AMCE potentielles réglementées par les Populations autochtones et communautés locales, auxquelles le principe de « consentement préalable, donné librement et en connaissance de cause " s'applique - confirmer l'intérêt pour l'autorité de gouvernance à faire évaluer la zone et à la faire signaler potentiellement en tant qu'AMCE.

2. Lire attentivement les lignes directrices et les critères de présélection, en discuter, et réunir une équipe d'examen composée de personnes connaissant la diversité des approches prises à l'échelle concernée pour la conservation par zone à cet endroit.

3. Avant d'appliquer l'outil de présélection (Section 3.2), compiler un ensemble complet de cartes et d'informations sur les emplacements potentiellement admissibles en tant qu'AMCE, après les avoir comparés par rapport aux cartes des aires protégées connues (désignées ou proposées), pour que la relation soit facilement comprise.

4. Appliquer chacun des quatre tests de présélection à chaque zone évaluée en tant qu'AMCE.

5. Identifier les zones qui réussissent les quatre tests en tant qu'AMCE candidates, et les évaluer à l'aide d'un outil d'évaluation empirique et adapté à l'échelle nationale (Section 3.3).

6. Établir un rapport destiné à la WDPA pour les AMCE qui réussissent le processus d'évaluation (Section 4).

7. Pour les zones qui ne réussissent pas les tests, noter les raisons des décisions pour chaque critère. Ces informations peuvent être utiles pour déterminer si des changements en matière de gouvernance ou de gestion pourraient permettre à la zone d'être admissible en tant qu'AMCE. Le cas échéant, appliquer de nouveau les points 1 à 5 ci-dessus. 


\subsection{Application de l'outil de présélection}

Cette section fournit des orientations sur la manière d'appliquer l'outil de présélection. Toutes les références aux " éléments " se réfèrent aux éléments de la définition, décrits à la Section 2.2.

Test 1. S'assurer que la zone n'est pas déjà reconnue et/ou enregistrée en tant qu'aire protégée.

La zone n'a pas déjà été reconnue ou proposée en tant qu'aire marine, d'eau douce ou terrestre protégée (voir élément a).

Test 2. S'assurer que la zone présente les caractéristiques essentielles définies pour être une AMCE.

1. Lieu : La zone doit être un espace géographiquement délimité. Les mesures de plus grande envergure pour les espèces et/ou l'environnement qui ne sont pas « par zone " échouent à ce test. Par exemple, les réglementations et interdictions relatives à la chasse au niveau national ou régional spécifiques à une espèce, les règles d'observation des baleines ou les fermetures temporaires de pêche (voir élément b) sont des mesures régionales spécifiques à une espèce et non de conservation in situ par zone.

2. Gouvernance et gestion durables : La zone est réglementée et gérée, et les dispositions attendues à ce titre devraient être continues et durables à long terme. II devrait y avoir un lien de causalité direct entre : (i) la gouvernance globale de la zone, son (ou ses) objectif(s) et sa gestion, et (ii) la conservation in situ de la biodiversité à long terme. Les zones où il n'y a ni autorité de gouvernance ni gestion ne sont pas des AMCE (voir éléments $c$, $d$ et f). Par conséquent, une zone actuellement à l'état naturel ou quasi naturel n'est pas automatiquement une AMCE.

3. Conservation in situ efficace de la biodiversité : La zone aboutit à la conservation in situ efficace de la biodiversité, avec des fonctions et services écosystémiques connexes. II devrait être clairement entendu que la zone conserve efficacement la biodiversité autochtone et les processus écosystémiques qui soutiennent la biodiversité. Cela peut être réalisé par divers modes de gouvernance et pratiques de gestion, y compris ceux associés à des valeurs culturelles, spirituelles, socioéconomiques et d'autres valeurs pertinentes localement. Les zones qui produisent des résultats pour la conservation seulement à court terme ou les zones qui ont vocation ou offrent un potentiel de conservation de la nature mais qui n'ont pas encore produit de résultats pour la conservation ne sont pas admissibles en tant qu'AMCE (voir éléments e, $g, h$, $i$ et $\jmath$ )..

4. La zone est exempte d'activités portant préjudice à l'environnement, et les menaces pour la biodiversité peuvent être gérées dans le cadre des systèmes de gouvernance et de gestion existants.

Test 3. S'assurer que le résultat pour la conservation perdurera à long terme.

Cela fait référence à la probabilité que le résultat pour la conservation perdure à long terme par tout moyen efficace, juridique ou autre (comme les lois coutumières ou des accords officiels avec les propriétaires fonciers, voir éléments e et f f. Ce test souligne la différence entre les efforts de conservation actuels, qui peuvent facilement s'inverser, et une AMCE qui peut produire des résultats de conservation durables à long terme

Test 4. S'assurer qu'un objectif de conservation in situ par zone (par exemple, l'Objectif 11 d'Aichi), par opposition à un objectif d'utilisation durable, est la bonne priorité pour l'établissement d'un rapport.

La conservation in situ de la biodiversité est l'un des trois objectifs primaires de la CDB. Dans le contexte de l'établissement de rapports à l'attention de la $\mathrm{CDB}$, au titre des Objectifs d'Aichi pour la biodiversité d'ici 2020, les aires protégées et les AMCE sont les principaux moyens d'aboutir à la conservation in situ et la principale priorité de l'Objectif 11 d'Aichi. Comme il est précisé dans l'Encadré 6, des mesures par zone peuvent également être appliquées pour aboutir à une utilisation durable des composantes de la biodiversité (par exemple, la priorité de l'Objectif 6 d'Aichi est la pêche durable et celui de l'Objectif 7 d'Aichi est l'agriculture, l'aquaculture et la sylviculture durables). II est cependant important de ne pas confondre ces mesures avec des mesures de conservation in situ pertinentes pour l'Objectif 11 d'Aichi. Au-delà de 2020, il restera important de rendre compte des mesures de conservation in situ (aires protégées et AMCE) par rapport à leurs objectifs appropriés, et des mesures d'utilisation durable par rapport aux leurs propres objectifs. Voir l'Annexe I sur la relation existant entre l'Objectif 11 et les autres Objectifs d'Aichi, ainsi que l'Annexe II pour consulter un arbre décisionnel sur la sélection de l'Objectif d'Aichi le plus approprié pour une mesure de conservation donnée.

\section{Les zones qui réussissent les quatre tests peuvent être} considérées en tant qu'AMCE candidates.

\section{3 Évaluation}

Les zones considérées en tant qu'AMCE candidates devraient ensuite faire l'objet d'un examen plus détaillé réalisé au cas par cas et impliquant des preuves empiriques. La Méthodologie d'évaluation des AMCE peut être téléchargée à partir du lien suivant : https://www.iucn.org/theme/protected-areas/wcpa/ what-we-do/oecms

Seules les zones passant cette évaluation empirique avec succès, avec la participation et le consentement effectif et entier de l'autorité de gouvernance, devront être signalées à la WDPA (voir Section 4 et Annexe III).

\subsection{Exemples d'autres mesures de conservation efficaces par zone (AMCE) potentielles}

Les situations suivantes peuvent être considérées en tant qu'AMCE potentielles. Ces exemples couvrent les différents modes de gouvernance aux fins d'illustrer leur applicabilité. Un certain nombre d'exemples dans lesquels la citation est marquée d'un astérisque (*) figurent dans un Numéro spécial de 


\section{Vérification de la pertinence de l'Objectif 11 d'Aichi}

Le Plan stratégique pour la diversité biologique 2011-2020 et les 20 Objectifs d'Aichi pour la biodiversité exigent un ensemble complet d'approches pour endiguer l'appauvrissement de la diversité biologique, y compris la sensibilisation à la biodiversité, l'élimination des incitations perverses pour sa dégradation, la mise en œuvre de plans de production durables, la réduction de l'appauvrissement des habitats, la prévention de l'extinction des espèces, la réduction des pressions directes sur la biodiversité à des niveaux durables, et la conservation de biodiversité in situ.

Les mesures de conservation par zone peuvent contribuer à la réalisation de plusieurs Objectifs d'Aichi, mais les mesures par zone ne parviennent pas toutes à atteindre leurs objectifs grâce à la conservation in situ de la biodiversité, conformément aux critères de l'Objectif 11.

Par exemple, de nombreuses fermetures de pêche s'appliquent à des étendues géographiques spécifiques et sont donc des mesures par zone, mais ces fermetures peuvent ne concerner que des espèces de poissons commerciaux spécifiques en diminution, ou bien s'appliquer à l'utilisation de certains types d'engins non sélectifs ou nuisibles aux habitats, ou encore n'être en vigueur qu'à certains moments de l'année lorsque des espèces vulnérables sont présentes à un stade de vie vulnérable (par exemple, en période de frai). Elles peuvent autoriser la poursuite d'activités en lien ou non avec la pêche (essais sismiques, forage pétrolier, etc.), tant que ces activités ne compromettent pas les raisons pour lesquelles ces fermetures ont été prévues. Ainsi, elles peuvent être des outils efficaces pour aider à garantir une gestion durable de la pêche (Objectif 6 d'Aichi), sans pour autant aboutir à la conservation in situ de la biodiversité (Objectif 11 d'Aichi).

De même, les plans de gestion forestière sont appliqués par zone et peuvent varier en termes d'impact écologique. Les approches à plus faible impact peuvent conserver plus d'espèces, de structures d'habitat et de fonctions écosystémiques que les approches à impact plus élevé, et certaines peuvent même conduire à une « utilisation durable » selon la définition de la CDB - c'est-à-dire «l'utilisation des éléments constitutifs de la diversité biologique d'une manière et à un rythme qui n'entraînent pas leur appauvrissement à long terme ". Cependant, leurs impacts sont de nature extractive et altèrent les écosystèmes, et par conséquent ces approches ne peuvent pas non plus aboutir à la conservation in situ de toute la biodiversité. II serait plus adapté de considérer ces mesures en tant que contributions à l'Objectif 7 d'Aichi, qui appelle à une gestion durable des zones consacrées à la sylviculture d'ici 2020.

La limite entre une mesure visant l'Objectif 7 et une mesure visant l'Objectif 11 peut être difficile à déterminer en cas d'utilisation coutumière des ressources biologiques dans des milieux largement naturels par les Populations autochtones et communautés locales. Dans de tels cas, afin de déterminer si une zone est une AMCE, il peut être utile d'étudier dans quelle mesure ces zones sont bien protégées contre les menaces forestières et non forestières à long terme.

Les autres Objectifs d'Aichi pour lesquels des mesures par zone peuvent fréquemment être utilisées comprennent l'Objectif 10 (réduire au minimum les nombreuses pressions anthropiques sur les récifs coralliens), l'Objectif 12 (éviter l'extinction des espèces menacées et améliorer leur état de conservation), l'Objectif 14 (restaurer et sauvegarder les écosystèmes qui fournissent des services essentiels), et l'Objectif 15 (conserver et restaurer les écosystèmes dégradés). Lorsque de telles mesures atteignent leurs objectifs par le biais de la conservation in situ de la biodiversité à long terme, elles peuvent également contribuer à l'Objectif 11. la revue PARKS sur les AMCE (IUCN/WCPA, 2018). https://doi. org/10.2305/IUCN.CH.2018.PARKS-24-SI.en.

\section{Conservation primaire}

Un site qui a un objectif de conservation primaire et qui aboutit à la conservation efficace de la biodiversité mais qui n'est pas signalé en tant qu'aire protégée pourrait être reconnu en tant qu'AMCE si l'autorité de gouvernance le souhaite.

\section{Voici quelques exemples :}

- Certains territoires ou aires (marines, terrestres ou d'eau douce) réglementés par les Populations autochtones, les communautés locales ou des entités privées, qui ont un objectif de conservation primaire et explicite, et qui aboutissent à la conservation in situ de la biodiversité, mais où l'organe de direction souhaite que ces territoires ou aires soient reconnus et signalés en tant qu'AMCE, plutôt qu'en tant qu'aires protégées.

- Les aires conservées privées, qui sont gérées avec un objectif de conservation spécifique mais qui ne sont pas reconnues en tant qu'aires protégées en vertu de la législation nationale (Mitchell et al., 2018), par exemple les aires de restauration écosystémiques en Indonésie (Utomo \& Walsh, 2018*).

- Les zones incluant des Zones clés pour la biodiversité, gérées de manière à aboutir à la conservation in situ de la biodiversité à long terme par le biais, notamment, de la réglementation ou d'autres approches efficaces.

- Certaines zones de mise en réserve permanente d'une forêt gérée, comme les forêts anciennes, les forêts primaires ou d'autres forêts à forte valeur de biodiversité, qui sont protégées contre les menaces forestières et non forestières.

- Certains espaces naturels gérés par les universités pour la recherche biologique.

\section{Conservation secondaire}

\section{Voici quelques exemples :}

- Les territoires et zones gérés par les Populations autochtones et/ou communautés locales (APAC, en intégralité ou en partie) pour préserver les écosystèmes naturels ou quasi naturels, avec 
une faible utilisation des ressources naturelles, pratiquée de manière durable et en veillant à ne pas dégrader la biodiversité de la zone. Cela comprend les aires côtières et marines, dans lesquelles les pratiques communautaires locales en matière de prélèvement et de gestion aboutissent de facto à la conservation des populations de poissons, des habitats et de la biodiversité marine associée, comme certaines aires marines gérées localement (LMMA) (Jupiter et al., 2014).

- Les systèmes de gestion traditionnels qui préservent un haut niveau de biodiversité associée. Cela pourrait inclure certains systèmes de gestion agricole ou forestière qui préservent les espèces autochtones et leur habitat (par exemple, Eghenter, 2018 ; Mwamidi et al., 2018*).

- Les parcs urbains ou municipaux gérés principalement pour les loisirs publics, mais qui sont assez vastes et suffisamment naturels pour permettre aussi d'aboutir à la conservation in situ efficace de la biodiversité (prairies sauvages, zones humides, etc.), et qui sont gérés pour maintenir ces valeurs de biodiversité (par exemple, Gray et al., 2018).

- Les terrains et eaux militaires, en intégralité ou en partie, dont la gestion est réalisée principalement à des fins de défense, mais dont les objectifs secondaires spécifiques sont axés sur la conservation de la biodiversité. La Base des Forces canadiennes Shilo, située dans l'écosystème de prairies mixtes du centre-sud du Manitoba (Canada), a été proposée par le Canada en tant qu'AMCE en 2019.

- Les bassins versants ou d'autres aires gérées principalement à des fins de gestion des ressources en eau, et qui aboutissent aussi à la conservation in situ de la biodiversité. Cela peut inclure, par exemple, les prairies inondables, les forêts fluviales, les forêts côtières, les zones humides, les rivières, les bassins versants en hauteur, ou d'autres zones gérées pour la stabilisation des sols et des pentes à long terme, l'atténuation des inondations, ou d'autres services écosystémiques (par exemple, Matallana-Tobón et al., 2018*).

- Les zones de fermeture de pêche permanente ou à long terme conçues pour protéger des écosystèmes complets en vue du recrutement des stocks, pour protéger les écosystèmes spécialisés dans leur intégralité, ou pour protéger les espèces à risque, grâce à la conservation in situ de la biodiversité dans son ensemble, et dont l'efficacité contre les menaces liées ou non à la pêche a été démontrée.

- Les réserves de chasse qui préservent les habitats naturels et d'autres espèces de faune et de flore, ainsi que des populations viables d'espèces autochtones chassées ou non.

- Les zones restaurées avec succès à partir d'écosystèmes dégradés ou menacés, pour fournir d'importants services écosystémiques, et qui contribuent également à la conservation efficace de la biodiversité, par exemple les zones humides côtières et en eau douce, restaurées à des fins de protection contre les inondations.

- Les zones qui contribuent à la conservation en raison de leur rôle pour relier les aires protégées et d'autres zones particulièrement importantes pour la conservation de la biodiversité, contribuant ainsi à la viabilité à long terme d'écosystèmes plus vastes (par exemple, Waithaka \& Warigia Njoroge, 2018*).

\section{Conservation dérivée}

\section{Voici quelques exemples :}

- Les sites naturels sacrés à forte valeur de biodiversité, qui sont conservés à long terme pour leur association avec un ou plusieurs groupes religieux (par exemple, Matallana-Tobón et al., 2018*).

- Les aires marines et côtières protégées pour des raisons autres que la conservation, mais qui aboutissent néanmoins à la conservation in situ de la biodiversité, par exemple les épaves historiques, les cimetières militaires sous-marins, etc. (voir Encadré 3).

- Les terrains et eaux militaires, en intégralité ou en partie, dont la gestion est réalisée à des fins de défense, mais qui aboutissent néanmoins à la conservation efficace de la biodiversité à long terme, même s'ils n'ont pas d'objectif secondaire.

\subsection{Exemples de zones peu susceptibles de répondre aux critères}

Il est peu probable que les zones et régimes de gestion suivants soient admissibles en tant qu'AMCE :

- Les petites zones semi-naturelles dans un paysage terrestre géré de manière intensive, avec une valeur limitée pour la conservation de la biodiversité, comme les parcs municipaux, les jardins classiques/privés, les arboretums, les lisières de champs, les bas-côtés des routes, les haies, les rivages étroits ou les marges de retrait des cours d'eau, les coupe-feu, les plages récréatives, les marinas et les terrains de golf.

- Les forêts gérées commercialement pour l'approvisionnement en bois et destinées à l'exploitation forestière, même si elles peuvent avoir des valeurs de conservation et soutenir certaines espèces d'intérêt. Ces zones devraient être considérées comme contribuant à l'Objectif 7 d'Aichi.

- Les fermetures de pêche et autres outils de gestion de l'espace halieutique, y compris, mais sans s'y limiter, les quotas de pêche ou les limitations de prises, les zones de mise en réserve temporaire ou les zones de restriction des engins dont la priorité est une seule espèce, un seul groupe d'espèces ou un seul habitat, qui peuvent faire l'objet d'une exploitation périodique et/ou être définis à des fins de gestion des stocks, et qui n'aboutissent pas à la conservation in situ des espèces, habitats et écosystèmes associés aux espèces ciblées. Ces zones devraient être considérées comme contribuant à l'Objectif 6 d'Aichi.

- Les terres agricoles gérées d'une manière limitant la conservation in situ de la biodiversité. Cela peut inclure, par exemple, les zones au pâturage trop intense pour soutenir les écosystèmes ou les espèces autochtones des prairies, ou bien les prairies replantées avec des monocultures ou des espèces non autochtones aux fins de la production animale.

- Les terres agricoles mises hors culture temporairement, les jachères estivales et les pratiques agricoles modifiées par subvention, qui pourraient profiter à la biodiversité. 
- Les mesures de conservation s'appliquant à une seule espèce ou à un seul groupe d'espèces, sur une vaste étendue géographique, comme les réglementations relatives à la chasse ou les règles d'observation des baleines, sont mieux considérées comme faisant partie d'un plus grand ensemble de mesures de conservation des espèces (Objectifs 5, 6, 7 et/ ou 12).

Les exemples ci-dessus n'ont pas vocation à être exhaustifs ou sans exception, mais ils visent à indiquer les types de zones pouvant être admissibles ou non en tant qu'AMCE. Lors de l'examen d'une zone, la conformité avec les définitions et critères appliqués au cours des quatre tests de présélection sera le meilleur moyen de garantir une identification homogène des AMCE candidates. Compte tenu de la diversité des situations dans lesquelles des AMCE peuvent exister, il est essentiel que toutes les zones considérées soient examinées soigneusement pour évaluer chaque cas spécifique.

Le concept d'AMCE sera généralement utilisé pour reconnaître les exemples existants de conservation efficace par zone et les régimes de gouvernance et de gestion qui les soutiennent. Le concept pourrait toutefois être également utilisé pour promouvoir de nouveaux efforts de conservation. Lors de la négociation relative à la Décision 14/8, des termes répondant aux critères d'admissibilité comme « ont une valeur importante pour la diversité biologique, ou comprennent des objectifs pour y parvenir » et " obtient ou obtiendra des résultats positifs durables pour la conservation in situ de la diversité biologique " ont été rajoutés au texte directeur pour le recensement des AMCE. Les gouvernements qui ont proposé ces ajouts ont souligné qu'ils serviraient dans le cas des sites où une restauration avait lieu, afin de reconnaître les tentatives de rétablissement écosystémique intentionnelles. Vouloir restaurer les écosystèmes et les habitats est louable, mais les zones de restauration ne devraient pas être reconnues en tant qu'AMCE tout le temps qu'elles n'auront pas produit de résultats tangibles et significatifs pour la biodiversité (voir Encadré 7 ).

\subsection{Droits et responsabilités des autorités de gouvernance}

Il existe de nombreuses raisons qui conduiront une autorité de gouvernance à envisager la reconnaissance de leur zone en tant qu'AMCE. Les autorités de gouvernance peuvent recenser une zone en tant qu'éventuelle AMCE et soit l'évaluer elles-mêmes soit demander un soutien indépendant pour déterminer si la zone est admissible en tant qu'AMCE d'après les présentes lignes directrices. Elles ont le droit de s'opposer à la reconnaissance ou désignation externe de leur zone en tant qu'AMCE lorsqu'elles n'ont pas donné leur consentement. Cela s'applique aux quatre modes de gouvernance définis plus haut (voir élément $c$ ). Lorsqu'une zone est reconnue en tant qu'AMCE, cela impose une responsabilité accrue à l'autorité de gouvernance, laquelle doit continuer de réglementer et de gérer la zone d'une manière aboutissant à la conservation in situ de la biodiversité.

\section{Encadré 7}

\section{Restauration écologique dans les AMCE}

La restauration écologique est le processus de gestion ou d'aide au rétablissement d'un écosystème dégradé, endommagé ou détruit, comme moyen de maintenir la résilience écosystémique et de conserver la biodiversité (CBD, 2016). II deviendra probablement un outil de conservation plus commun et nécessaire à l'avenir.

Les zones faisant, ou proposées pour faire, l'objet d'efforts de restauration actifs, ne devraient pas être reconnues en tant qu'AMCE tout le temps qu'elles n'auront pas produit de résultats tangibles et significatifs pour la biodiversité. Les orientations de I'UICN sont donc que les zones de restauration proposées en tant qu'AMCE doivent remplir toutes les conditions suivantes :

1. La restauration se déroule dans un écosystème à forte valeur de biodiversité (voir Encadré 4) de manière à ce que la zone, une fois restaurée, soit admissible en tant qu'AMCE en raison de sa valeur de conservation et de sa contribution au renforcement des réseaux d'aires protégées existants ;

2. Tout effort de restauration devrait (i) avoir réduit les menaces qui ont causé la dégradation initiale et l'appauvrissement de la biodiversité, (ii) démontrer un rétablissement écosystémique réussi, fondé sur les principes de la restauration écologique, et (iii) contribuer au maintien à long terme d'un écosystème résilient et en évolution ; et

3. Démonstration d'un type de restauration écologique active ou de régénération naturelle à une échelle qui devrait retrouver et maintenir une intégrité écologique et un ensemble complet d'espèces.

\subsection{Soutien aux AMCE}

La reconnaissance d'autres mesures de conservation efficaces par zone devrait être soutenue par des mesures visant à renforcer la capacité de gouvernance de leurs autorités légitimes et à garantir des résultats positifs et durables pour la biodiversité. Même si les circonstances nationales diffèrent, toute législation connexe devrait apporter un soutien et une reconnaissance plus importants aux systèmes de gouvernance existants et ne pas chercher à supplanter ou modifier inutilement les dispositions locales qui sont efficaces. 


\section{Suivi des autres mesures de conservation efficaces par zone et établissement de rapports à leur sujet}

\begin{abstract}
Il est nécessaire de surveiller l'efficacité des AMCE. Cela devrait inclure : (i) une documentation de base et le suivi continu des valeurs de biodiversité des sites ; (ii) le suivi communautaire continu, la cartographie participative et l'intégration des connaissances traditionnelles, le cas échéant ; (iii) le suivi des actions de conservation, y compris celles axées sur le maintien de la biodiversité et l'amélioration de la conservation in situ ; et (iv) le suivi de la gouvernance, de la participation des intervenants et des systèmes de gestion qui contribuent aux résultats pour la biodiversité (Haase et al., 2018 ; Woodley et al., 2015).
\end{abstract}

Un élément clé de la définition d'une AMCE est qu'elle doit être "réglementée et gérée de façon à obtenir des résultats positifs et durables à long terme pour la conservation (...) de la diversité biologique ». Ceci est lié au concept d'efficacité de la gestion. Par conséquent, le suivi et l'établissement de rapports sur l'efficacité des AMCE seront essentiels pour veiller à ce que les sites continuent de produire des résultats en matière de conservation (Woodley et al., 2015). La mesure de l'Efficacité de la gestion des aires protégées (EGAP) sera, dans de nombreux cas, le moyen le plus pragmatique de mesurer l'efficacité des AMCE, mais les outils d'EGAP devraient s'appuyer sur des informations quantitatives supplémentaires concernant les résultats pour la biodiversité. L'utilisation de la norme de la Liste verte des aires protégées et conservées de l'UICN viendra également compléter cette documentation (IUCN, 2017). Les autorités chargées des AMCE devraient s'assurer qu'un suivi adéquat de l'efficacité de la gestion est mis en place afin de permettre aux résultats pour la conservation de perdurer à long terme (voir Hockings et al., 2015). Ces informations devraient également être communiquées au PNUE-WCMC pour intégration dans la Base de données mondiale sur l'efficacité de la gestion des aires protégées (GD-PAME, Global Database on Protected Area Management Effectiveness).

Le concept d'" autres mesures de conservation efficaces par zone » est le fruit de décisions prises par les Parties à la CDB. Lors de l'adoption de la définition des AMCE, la CdP 14 de la CDB a également encouragé les parties à soumettre des données sur les AMCE à la base de données mondiale sur Les Aires protégées (WDPA) gérée par le PNUE-WCMC (CBD, 2018). Afin de remplir cette obligation, le PNUE-WCMC a créé une base de données parallèle pour les AMCE dans le cadre de l'initiative Protected Planet, en complément de la WDPA. L'initiative Protected Planet englobe plusieurs bases de données accessibles et téléchargeables sur son site Web www.protectedplanet.net. Le PNUE-WCMC utilise les données figurant dans ces bases pour mesurer les progrès enregistrés par rapport aux objectifs de conservation internationaux, comme l'Objectif 11 d'Aichi pour la biodiversité et les ODD 14 et 15.

Les mesures par zone qui sont admissibles en tant qu'aires protégées ou en tant qu'AMCE devraient être signalées pour intégration, respectivement, dans la WDPA ou la base de données des AMCE. Ces signalements (établissement de rapports) devraient être effectués avec le consentement préalable, donné librement et en connaissance de cause des autorités de gouvernance correspondantes.

Pour plus d'informations sur les exigences en matière d'établissement de rapports destinés à la WDPA et à la base de données des AMCE, et sur la vérification des données, voir le Tableau 1 ci-après, l'Annexe III et les orientations disponibles sur www.wcmc.io/oecm_guidance.

Tableau 1. Principes de base pour la vérification des données à inclure dans les bases de données de Protected Planet

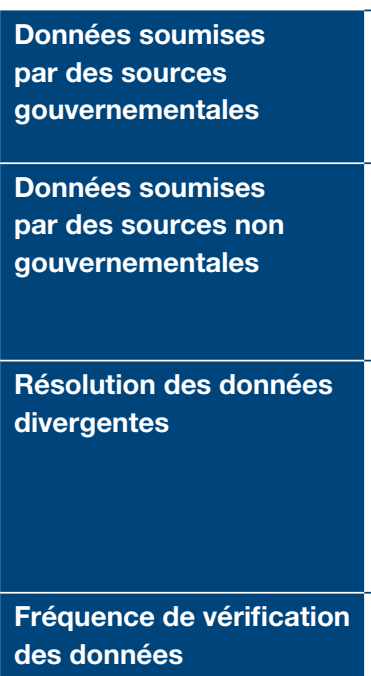

Conformément aux mandats officiels relatifs à la WDPA, les données soumises par des sources gouvernementales concernant les aires protégées ou les AMCE seront considérées comme ayant été vérifiées par l'État et seront incluses dans la WDPA et la base de données des AMCE après mise en forme et contrôle de la qualité des données.

Les données entrantes provenant de fournisseurs non gouvernementaux sont soumises à un processus de vérification avant d'être ajoutées aux bases de données de Protected Planet.

Les données peuvent être vérifiées soit par des vérificateurs d'État, soit par des vérificateurs experts. Si aucune des parties ne peut vérifier les données, elles ne seront pas intégrées dans les bases de données de Protected Planet.

En cas de divergence d'opinions entre le fournisseur de données et le vérificateur des données (par exemple, en cas de différend sur les délimitations correctes d'un site), cela sera discuté avec les deux parties dans le but de parvenir à une solution.

Les fournisseurs de données sont informés du processus de vérification lors de la soumission des données et sont tenus informés de l'avancement. Dans les cas où aucune résolution n'est trouvée, les données ne pourront pas être intégrées dans les bases de données de Protected Planet.

Le PNUE-WCMC s'efforce d'actualiser toutes les données au moins une fois tous les cinq ans.

Pour toute question concernant l'établissement de rapports, veuillez contacter : protectedareas@unep-wcmc.org 


\section{Références}

Borrini-Feyerabend, G., Dudley, N., Jaeger, T., Lassen, B., Pathak Broome, N., Phillips, A. and Sandwith, T. (2014). Gouvernance des aires protégées : de la compréhension à l'action. Lignes Directrices des meilleures pratiques pour les aires protégées №20. Gland, Switzerland: IUCN. https://portals.iucn.org/library/ node/44864

Borrini-Feyerabend, G. and Hill, R. (2015). 'Governance for the conservation of nature', in Worboys, G. L., Lockwood, M., Kothari, A., Feary, S. and Pulsford, I. (eds) Protected Area Governance and Management, pp. 169-206. ANU Press: Canberra.

Canada Department of Fisheries and Oceans (2016). Operational guidance for identifying "other effective area-based conservation measures" in Canada's marine environment. Canada Department of Fisheries and Oceans: Ottawa, Canada. 9pp. Consulté le 17 janvier 2017 à : http://www.dfo-mpo.gc.ca/oceans/documents/ publications/oeabcm-amcepz/2016_11_24_OEABCM-marineguidance-for-public-audience_Version-1b.pdf

Convention on Biological Diversity (CBD) (1992). Convention on Biological Diversity. https://www.cbd.int/convention/text/

CBD (2010). Strategic Plan on Biodiversity 2011-2020. https:// www.cbd.int/sp/

CBD (2012). Decision on Protected Areas (XI/24). https://www. cbd.int/doc/decisions/cop-11/cop-11-dec-24-en.pdf

CBD (2016). Progress Towards the Achievement of Aichi Biodiversity Targets 11 and 12 (Decision XIII/2). https://www.cbd. int/doc/decisions/cop-13/cop-13-dec-02-en.pdf

CBD (2016). Ecosystem restoration: short-term action plan. CBD/ COP/DEC/XIII/5, 10 December 2016. https://www.cbd.int/doc/ decisions/cop-13/cop-13-dec-05-en.pdf

CBD (2018). Protected areas and other effective area-based conservation measures (Decision 14/8). https://www.cbd.int/doc/ decisions/cop-14/cop-14-dec-08-en.pdf

CBD (2019). Post-2020 Global Biodiversity Framework: Discussion Paper. https://www.cbd.int/doc/c/d431/ b38f/3d580bb73e7c2b5aaa286310/post2020-prep-01-01-en.pdf

Day, J., Dudley, N., Hockings, M., Holmes, G., Laffoley, D., Stolton, S. and Wells, S. (2012). Guidelines for applying the IUCN Protected Area Management Categories to Marine Protected Areas. IUCN: Gland, Switzerland.

Donald, P., Buchanan, G.M., Balmford, A., et al. (2019). 'The prevalence, characteristics and effectiveness of Aichi Target 11's "other effective area-based conservation measures" (OECMs) in Key Biodiversity Areas'. Conservation Letters.

2019;e12659. https://doi.org/10.1111/conl.12659

Dudley, N. (ed) (2008). Lignes directrices pour l'application des catégories de gestion aux aires protégées. IUCN: Gland, Switzerland. https://doi.org/10.2305/IUCN.CH.2008.PAPS.2.fr, https://portals.iucn.org/library/node/48887

Dunstan, P. K., Bax, N.J., Dambacher, J.M., Hayes, K.R., Hedge, P.T., Smith, D.C., and Smith, A.D.M. (2016). 'Using ecologically or biologically significant marine areas (EBSAs) to implement marine spatial planning'. Ocean \& Coastal Management, 121, 116-127.

Eghenter C. (2018). 'Indigenous effective area-based conservation measures: conservation practices among the Dayak Kenyah of North Kalimantan'. PARKS 24: 69-78.. IUCN: Gland.
Gray, M. (2004). Geodiversity: Valuing and Conserving Abiotic Nature. John Wiley and Sons: UK.

Gray, P.A., Cheriton, D., Gaetz, N., Lehman, P., Sherwood, J., Beechey, T. J. and Lemieux C.J. (2018). 'Comparing screening tools for assessment of potential 'other effective area-based conservation measures' in Ontario, Canada'. PARKS 24. IUCN: Gland.

Gross, J.E., Woodley, S., Welling, L.A., and Watson, J. (eds.) (2016). Adapting to Climate Change: Guidance for protected area managers and planners. Best Practice Protected Area Guidelines Series No. 24. IUCN: Gland, Switzerland. https://portals.iucn.org/ library/node/46685

Haase, P., Tonkin, J.D., Stoll, S., Burkhard, B., Frenzel, M., Geijzendorffer, I.L., Häuser, C. et al. (2018). 'The next generation of site-based long-term ecological monitoring: Linking essential biodiversity variables and ecosystem integrity'. Science of the Total Environment 613: 1376-1384.

Indigenous Circle of Experts, (2018). We Rise Together: Achieving Pathway to Canada Target 1 through the creation of Indigenous Protected and Conserved Areas in the spirit and practice of reconciliation. Indigenous Circle of Experts, Pathway to Canada Target 1.

Hockings, M., Leverington, F. and Cook, C. (2015). 'Protected area management effectiveness', in Worboys, G. L., Lockwood, M., Kothari A., Feary S. and Pulsford I. (eds) Protected Area Governance and Management. ANU Press, Canberra.

IUCN (2016). A Global Standard for the Identification of Key Biodiversity Areas. First edition. IUCN: Gland, Switzerland.

IUCN (2017). Green List of Protected and Conserved Areas Standard. https://www.iucn.org/theme/protected-areas/ourwork/iucn-green-list, https://portals.iucn.org/library/node/46259

IUCN/WCPA (2018). Special Issue on "Other Effective Areabased Conservation Measures". PARKS 24. IUCN: Gland. https:// doi.org/10.2305/IUCN.CH.2018.PARKS-24-SI.en

Jonas, H., Barbuto, V., Jonas, H.C., Kothari, A. and Nelson, F. (2014). 'New steps of change: looking beyond protected areas to consider other effective area based conservation measures'. PARKS 20 (2): 111-128. DOI: https://doi.org/10.2305/IUCN. CH.2014.PARKS-20-2.HDJ.en

Jonas, H. and MacKinnon, K. (eds) (2016). Co-Chairs' Report of the First Meeting of International Experts of the Task Force on Other Effective Area-based Conservation Measures. IUCNWCPA: Gland, Switzerland. https://www.iucn.org/theme/ protected-areas/wcpa/what-we-do/oecms

Jonas, H. and MacKinnon, K. (Eds) (2016). Advancing Guidance on Other Effective Area-based Conservation Measures: Report of the Second Meeting of the IUCN-WCPA Task Force on Other Effective Area-based Conservation Measures. IUCN : Gland, Switzerland https://www.iucn.org/theme/protected-areas/wcpa/ what-we-do/oecms

Jonas, H. et MacKinnon, K. (eds) (2017). Using Case Studies to Enhance Guidance on Other Effective Area-based Conservation Measures: Report of Third Meeting of the IUCN-WCPA Task Force on Other Effective Area-based Conservation Measures. IUCN-WCPA: Gland, Switzerland. https://www.iucn.org/theme/ protected-areas/wcpa/what-we-do/oecms 
Jonas, H. and Sandwith, T. (eds) (2019). Towards Recognising, Reporting and Supporting OECMs: Report of the Fourth Expert Meeting of the IUCN-WCPA Task Force on Other Effective Areabased Conservation Measures. IUCN: Gland, Switzerland https:// www.iucn.org/theme/protected-areas/wcpa/what-we-do/oecms

Jonas, H., MacKinnon, K., Dudley, N., Hockings, M., Jessen, S., Laffoley, D., MacKinnon, D., Matallana-Tobón, C., Sandwith, T., Waithaka J. and Woodley, S. (2018). 'Other effective area-based conservation measures: From Aichi Target 11 to the post-2020 Biodiversity Framework'. PARKS 24: 9-16 IUCN: Gland.

Jupiter, S., Cohen, P., Weeks, R., Tawake, A. and Govan, H. (2014). 'Locally-managed marine areas: Multiple objectives and diverse strategies'. Pacific Conservation Biology 20. 10.1071/ PC140165.

Laffoley, D., Dudley, N., Jonas, H., MacKinnon, D., MacKinnon, K., Hockings, M. and Woodley, S. (2017). 'An introduction to "other effective area-based conservation measures" under Aichi Target 11 of the Convention on Biological Diversity: origin, interpretation and some emerging ocean issues'. Journal of Aquatic Conservation 27 (Supplement 1): 130-137.

Leverington, F., Lemos Costa, K., Courrau, J., Pavese, H., Nolte, C., Marr, M., Coad, L., Burgess, N., Bomhard, B. and Hockings, M. (2010). Management effectiveness evaluation in protected areas - a global study, Second edition. The University of Queensland Brisbane, Australia.

Lopoukhine, N. and Dias, B.F. (2012). 'Editorial: What does Target 11 really mean?' PARKS 18 (1): 5-8.

MacKinnon, D., C.J. Lemieux, K. Beazley, S. Woodley, R. Helie, J. Perron, J. Elliott, C. Haas, J. Langlois, H. Lazaruk, T. Beechey, and P. Gray (2015). 'Canada and Aichi Biodiversity Target 11: understanding "other effective area-based conservation measures" in the context of the broader target'. Biodiversity and Conservation 24 (14): 3559-3581. DOI 10.1007/s10531-0151018-1.

Matallana-Tobón, C., Santamaría, M., Areiza Tapias, A., Solano C. and Galán S. (2018). 'Rethinking nature conservation in Colombia: a case study of other effective area-based conservation measures'. PARKS 24: 89-98. IUCN: Gland. https://doi. org/10.2305/IUCN.CH.2018.PARKS-24-SICLM.en

Mathur, V. B., Onial, M. and Mauvais, G. (2015) "Managing threats", in G. L. Worboys, M. Lockwood, A. Kothari, S. Feary and I. Pulsford (eds). Protected Area Governance and Management, pp. 473-494, ANU Press, Canberra.

Mitchell, B., Fitzsimons, J., Stevens, C. and Wright, D. (2018). 'PPA or OECM? Differentiating between privately protected areas and other effective area-based conservation measures on private land'. PARKS 24: 49-60. IUCN: Gland. https://doi.org/10.2305/ IUCN.CH.2018.PARKS-24-SIBAM.en

Mwamidi, D.M., Renom, J.G. Fernández-Llamazares, Á., Burgas, D., Domínguez, P. and Cabeza, M. (2018). 'Contemporary pastoral commons in East Africa as OECMs: a case study from the Daasanach community'. PARKS 24: 79-88. IUCN: Gland.

UNEP-WCMC and IUCN (2016). Protected Planet Report 2016. UNEP-WCMC and IUCN: Cambridge UK and Gland, Switzerland.

UNEP-WCMC (2017). World Database on Protected Areas User Manual 1.5. UNEP-WCMC: Cambridge, UK. Available at: http:// wcmc.io/WDPA_Manual
UNEP-WCMC and IUCN (2018). Protected Planet Report 2018. UNEP-WCMC and IUCN: Cambridge UK and Gland, Switzerland. https://portals.iucn.org/library/node/48344

UNEP-WCMC (2018). 2018 United Nations List of Protected Areas. Supplement on protected area management effectiveness. UNEP-WCMC: Cambridge, UK.

United Nations (2007). United Nations Declaration on the Rights of Indigenous Peoples. Available at: https://www.un.org/ development/desa/indigenouspeoples/wp-content/uploads/ sites/19/2018/11/UNDRIP_E_web.pdf

Utomo, A.B. and Walsh T.A. (2018). 'Hutan Harapan ecosystem restoration concession, Sumatra, Indonesia: a potential OECM?' PARKS 24: 61-68. IUCN: Gland. https://doi.org/10.2305/IUCN. CH.2018.PARKS-24-SIABU.en

Waithaka, J. and Warigia Njoroge, G. (2018). 'The role of potential OECMs in safeguarding space for nature in Kenya: A case study of wildlife conservancies'. PARKS 24: 99-106. IUCN: Gland.

Watson, James EM, Nigel Dudley, Daniel B. Segan, and Marc Hockings. 'The performance and potential of protected areas'. Nature 515, no. 7525 (2014): 67.

Woodley, S., Bertzky B., Crawhall, N., Dudley, N., Miranda Londoño, J., MacKinnon, K., Redford, K.R. and Sandwith, T. (2012). 'Meeting Aichi Target 11: What does success look like for protected area systems?' PARKS 18 (1): 23-36.

Woodley, S., MacKinnon, K., McCanny, S., Pither, R., Prior, K. Salafsky, N. and Lindenmayer, D. (2015). 'Managing protected areas for biological diversity and ecosystem functions', in Worboys, G.L., Lockwood, M., Kothari, A., Feary, S. and Pulsford, I. (eds.) Protected Area Governance and Management, pp. 651-684, ANU Press, Canberra. http://press.anu.edu.au/ wpcontent/uploads/2015/02/CHAPTER21.pdf

Zarnetske, P.L., Read, Q.R., Record, S., Gaddis, K.D., Pau, S., Hobi, M.L., Malone, S.L., Costanza, J., Dahlin K.M., Latimer A.M., Wilson, A.M., Grady, J.M., Ollinger, S.V. and Finley A.O. (2019). 'Towards connecting biodiversity and geodiversity across scales with satellite remote sensing'. Ecological Soundings. https://doi.org/10.1111/geb.12887 


\section{Annexe I}

\section{Relation entre les Objectifs d'Aichi et l'Objectif 11}

(Adaptation à partir de Laffoley et al., 2017).

\begin{tabular}{|c|c|c|}
\hline Objectif & Texte & Relation avec l'Objectif 11 (T11) \\
\hline T3 & $\begin{array}{l}\text { D'ici à } 2020 \text { au plus tard, les incitations, y compris } \\
\text { les subventions néfastes pour la diversité biologique, } \\
\text { sont éliminées, réduites progressivement ou } \\
\text { réformées, afin de réduire au minimum ou d'éviter } \\
\text { les impacts défavorables, et des incitations positives } \\
\text { en faveur de la conservation et de l'utilisation } \\
\text { durable de la diversité biologique sont élaborées } \\
\text { et appliquées, d'une manière compatible et en } \\
\text { harmonie avec les dispositions de la Convention } \\
\text { et les obligations internationales en vigueur, en } \\
\text { tenant compte des conditions socioéconomiques } \\
\text { nationales. }\end{array}$ & $\begin{array}{l}\text { Les incitations positives pour la conservation et l'utilisation } \\
\text { durable de la biodiversité qui entraînent la conservation in situ } \\
\text { de la nature par zone, telles que les incitations fiscales pour les } \\
\text { propriétaires d'aires conservées privées, sont des exemples } \\
\text { de mesures T3 (c.-à-d. pour l'Objectif 3) qui contribuent aussi } \\
\text { à la réalisation de l'Objectif } 11 \text {. }\end{array}$ \\
\hline $\mathrm{T} 4$ & $\begin{array}{l}\text { D'ici à } 2020 \text { au plus tard, les gouvernements, les } \\
\text { entreprises et les parties prenantes, à tous les } \\
\text { niveaux, ont pris des mesures ou ont appliqué } \\
\text { des plans pour assurer une production et une } \\
\text { consommation durables, et ont maintenu les } \\
\text { incidences de l'utilisation des ressources naturelles } \\
\text { dans des limites écologiques sûres. }\end{array}$ & $\begin{array}{l}\text { Les plans de production durable (mesures T4) peuvent } \\
\text { inclure des zones non exploitées mises en réserve en tant } \\
\text { que « police d'assurance » ou « source de semences ", qui } \\
\text { contribuent à assurer la durabilité de l'utilisation dans une } \\
\text { zone plus large. Lorsque ces zones mises en réserve sont } \\
\text { efficaces pour la conservation in situ de la biodiversité à long } \\
\text { terme, elles peuvent contribuer à l'Objectif } 11 .\end{array}$ \\
\hline T5 & $\begin{array}{l}\text { D'ici à 2020, le rythme d'appauvrissement de tous } \\
\text { les habitats naturels, y compris les forêts, est réduit } \\
\text { de moitié au moins et si possible ramené à près } \\
\text { de zéro, et la dégradation et la fragmentation des } \\
\text { habitats sont sensiblement réduites. }\end{array}$ & $\begin{array}{l}\text { La création de zones pour l'Objectif } 11 \text { (ci-après « zones T11 } \\
\text { ") est un moyen important pour la réalisation de l'Objectif } 5 \text {. } \\
\text { de la nature à long terme, qu'il s'agisse d'aires protégées } \\
\text { ou d'AMCE, peut prévenir l'appauvrissement des habitats } \\
\text { naturels ainsi que la dégradation et la fragmentation des } \\
\text { écosystèmes, surtout si ces zones sont bien gérées. Dans un } \\
\text { contexte terrestre, cela peut concerner les forêts primaires et, } \\
\text { dans un contexte marin, cela pourrait être particulièrement } \\
\text { valable dans le cas d'habitats tels que les récifs coralliens, les } \\
\text { herbiers marins et les monts sous-marins. }\end{array}$ \\
\hline T6 & $\begin{array}{l}\text { D'ici à 2020, tous les stocks de poissons et } \\
\text { d'invertébrés et plantes aquatiques sont gérés } \\
\text { et récoltés d'une manière durable, légale et } \\
\text { en appliquant des approches fondées sur les } \\
\text { écosystèmes, de telle sorte que la surpêche } \\
\text { soit évitée, que des plans et des mesures de } \\
\text { récupération soient en place pour toutes les espèces } \\
\text { épuisées, que les pêcheries n'aient pas d'impacts } \\
\text { négatifs marqués sur les espèces menacées et } \\
\text { les écosystèmes vulnérables, et que l'impact } \\
\text { de la pêche sur les stocks, les espèces et les } \\
\text { écosystèmes reste dans des limites écologiques } \\
\text { sûres. }\end{array}$ & $\begin{array}{l}\text { Les zones T11 peuvent contribuer à assurer la durabilité de } \\
\text { l'exploitation des éléments de la biodiversité dans l'ensemble } \\
\text { du paysage marin, ceci en fournissant : des repères par } \\
\text { rapport auxquels les effets des décisions de gestion peuvent } \\
\text { être évalués; des fonctions de « police d'assurance » et de } \\
\text { " source de semences " pour récupérer après des erreurs } \\
\text { de gestion ; et/ou des avantages indirects pour le paysage } \\
\text { marin dans son ensemble. Les mesures de conservation des } \\
\text { espèces ou habitats qui s'appliquent à des paysages marins } \\
\text { de plus vastes plutôt qu'à étendues géographiques distinctes } \\
\text { et bien délimitées, et qui ne sont pas en place à long terme, } \\
\text { devraient correspondre à l'Objectif } 6 \text {. L'utilisation durable des } \\
\text { ressources biologiques peut être un objectif pour certaines } \\
\text { zones T11. La grande différence entre les mesures T11 et T6 } \\
\text { par zone est que les zones T11 aboutissent à la conservation } \\
\text { in situ de la nature dans son ensemble, et ce résultat ne doit } \\
\text { pas être compromis par les utilisations autorisées. }\end{array}$ \\
\hline T7 & $\begin{array}{l}\text { D'ici à } 2020 \text {, les zones consacrées à l'agriculture, } \\
\text { l'aquaculture et la sylviculture sont gérées d'une } \\
\text { manière durable, afin d'assurer la conservation de la } \\
\text { diversité biologique. }\end{array}$ & $\begin{array}{l}\text { Les zones T11 intégrées dans des paysages terrestres } \\
\text { gérés principalement pour l'agriculture, l'aquaculture ou } \\
\text { la sylviculture peuvent aider à garantir que ces activités } \\
\text { ne provoquent pas d'appauvrissement irréversible de la } \\
\text { biodiversité dans des paysages terrestres plus vastes, ceci } \\
\text { en fournissant des repères par rapport auxquels les effets } \\
\text { des décisions de gestion peuvent être évalués. Elles peuvent } \\
\text { également avoir des fonctions de « police d'assurance » et } \\
\text { de « source de semences » pour récupérer après les erreurs } \\
\text { de gestion, et fournir des avantages indirects ainsi que des } \\
\text { contributions pour la connectivité dans le paysage terrestre } \\
\text { plus vaste. }\end{array}$ \\
\hline
\end{tabular}




\section{Objectif}

T9

T10

T12

T14

T15

T18

\section{Texte}

D'ici à 2020, les espèces exotiques envahissantes et les voies d'introduction sont identifiées et classées en ordre de priorité, les espèces prioritaires sont contrôlées ou éradiquées et des mesures sont en place pour gérer les voies de pénétration, afin d'empêcher l'introduction et l'établissement de ces espèces.

\section{D'ici à 2015, les nombreuses pressions anthropiques} exercées sur les récifs coralliens et les autres écosystèmes vulnérables marins et côtiers affectés par les changements climatiques ou l'acidification des océans sont réduites au minimum, afin de préserver leur intégrité et leur fonctionnement.

D'ici à 2020, l'extinction d'espèces menacées connues est évitée et leur état de conservation, en particulier de celles qui tombent le plus en déclin, est amélioré et maintenu.

\section{Relation avec l'Objectif 11 (T11)}

Les zones T11 ayant des objectifs de gestion pour maintenir ou restaurer l'intégrité écologique peuvent être une priorité pour les mesures T9 afin d'éliminer les espèces exotiques.

Les mesures T11 peuvent avoir de la valeur pour la protection des récifs coralliens et d'autres écosystèmes vulnérables face aux pressions anthropiques telles que la dégradation des habitats et la surexploitation des espèces. Toutefois, les mesures T11 ne peuvent, à elles seules, répondre pleinement aux menaces du changement climatique et de l'acidification des océans, ce qui nécessite une réduction des gaz à effet de serre à l'échelle mondiale.

Les mesures T11 sont un outil majeur pour prévenir l'extinction et faciliter le rétablissement des espèces menacées, grâce à une conservation in situ à long terme des espèces et de leurs écosystèmes associés. Les mesures T12 axées sur des espèces individuelles, ne s'appliquant pas par zone ni à long terme, ou qui ne sont pas réalisées par le biais de la conservation in situ de la biodiversité dans son ensemble, ne sont pas non plus des mesures T11. Les mesures T11 peuvent prévenir l'extinction et faciliter le rétablissement des espèces menacées, contribuant ainsi à l'Objectif 12 .

Les mesures T11 peuvent être un moyen de contribuer à la réalisation de l'Objectif 14, en protégeant les écosystèmes qui fournissent une variété de services. Certaines mesures T14 peuvent également être reconnues comme contribuant à l'Objectif 11 si elles sont réalisées par le biais de la conservation in situ de la biodiversité à long terme, indépendamment de leurs objectifs primaires. Dans un contexte marin, cela pourrait être la préservation des récifs coralliens ou des mangroves dans le cadre, par exemple, de la protection côtière contre les tempêtes et les ondes océaniques. bien-être, sont restaurés et sauvegardés, compte tenu des besoins des femmes, des communautés autochtones et locales, et des populations pauvres et vulnérables.

En raison de leurs niveaux d'intégrité écologique généralement plus élevés que les paysages terrestres et marins exploités, les zones $\mathrm{T} 11$ sont souvent plus résilientes et plus diverses, et elles stockent davantage de carbone. La protection des zones intactes ainsi que la protection et la restauration des aires dégradées sont deux manières dont les mesures T11 peuvent contribuer à l'Objectif 15. Les mesures T15 qui réalisent leurs objectifs par le biais de la conservation in situ de la biodiversité à long terme peuvent être reconnues en tant que zones T11. restauration d'au moins $15 \%$ des écosystèmes dégradés, contribuant ainsi à l'atténuation des changements climatiques et l'adaptation à ceux-ci, ainsi qu'à la lutte contre la désertification.

D'ici à 2020, les connaissances, innovations et pratiques traditionnelles des communautés autochtones et locales qui présentent un intérêt pour la conservation et l'utilisation durable de la diversité biologique, ainsi que leur utilisation coutumière durable, sont respectées, sous réserve des dispositions de la législation nationale et des obligations internationales en vigueur, et sont pleinement intégrées et prises en compte dans le cadre de l'application de la Convention, avec la participation entière et effective des communautés autochtones et locales, à tous les niveaux pertinents.

\section{Les mesures T11 peuvent contribuer à l'Objectif 18 en aidant} à garantir que les zones où se sont développés le savoir traditionnel, les innovations et les pratiques des communautés autochtones et locales, ainsi que leur utilisation coutumière des ressources biologiques, restent intactes écologiquement et capables de soutenir ces activités à long terme. À l'inverse, certaines zones indigènes gérées traditionnellement peuvent contribuer à l'Objectif 11, par exemple certains sites naturels sacrés n'appartenant pas au réseau d'aires protégées officiel. 


\section{Annexe II}

\section{Aide à la décision : L'Objectif 11 est-il le plus pertinent des Objectifs d'Aichi pour évaluer une mesure de conservation?}

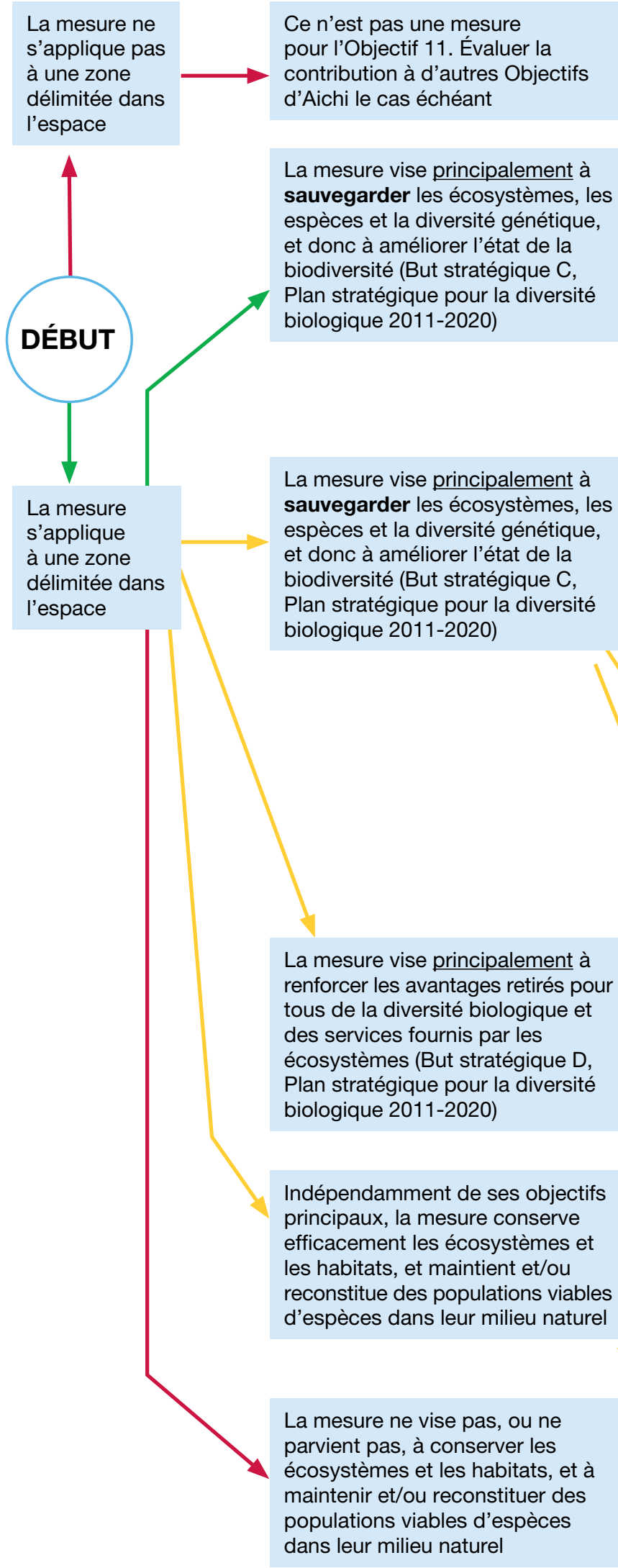

La mesure est déjà ou pourrait être reconnue en tant qu'aire protégée

La mesure vise principalement à conserver les écosystèmes et les habitats, et à maintenir et/ou reconstituer des populations viables d'espèces dans leur milieu naturel (AMCE potentielle pour l'Objectif 11)

La mesure vise principalement à éviter l'extinction des espèces menacées connues, et à améliorer et préserver leur état de conservation (mesure potentielle pour l'Objectif 12)

La mesure vise principalement à maintenir la diversité génétique des plantes cultivées et des animaux d'élevage ou domestiques, et celle des espèces sauvages apparentées, y compris d'autres espèces à valeur socioéconomique et culturelle (mesure potentielle pour l'Objectif 13)

La mesure vise principalement à réduire ou à mettre un terme à l'appauvrissement, la dégradation et la fragmentation des habitats naturels (mesure potentielle pour l'Objectif 5)

La mesure vise principalement à s'assurer que les stocks de poissons, invertébrés et plantes aquatiques sont gérés et récoltés durablement (mesure potentielle pour l'Objectif 6)

La mesure vise principalement à s'assurer que les zones consacrées à l'agriculture, l'aquaculture et la sylviculture sont gérées durablement (mesure potentielle pour l'Objectif 7)

La mesure vise principalement à réduire au minimum les nombreuses pressions anthropiques exercées sur les récifs coralliens et les autres écosystèmes vulnérables marins et côtiers affectés par les changements climatiques ou l'acidification des océans, afin de préserver leur intégrité et leur fonctionnement (mesure potentielle pour l'Objectif 10)

La mesure vise principalement à restaurer et à sauvegarder les écosystèmes qui fournissent des services essentiels, en particulier l'eau et contribuent à la santé, aux moyens de subsistance et au bien-être (mesure potentielle pour l'Objectif 14)

La mesure vise principalement à améliorer la résilience des écosystèmes et la contribution de la diversité biologique aux stocks de carbone grâce aux mesures de conservation et restauration, y compris la restauration des écosystèmes dégradés (mesure potentielle pour l'Objectif 15)

Évaluer en tant qu'AMCE potentielle pour l'Objectif 11 et la contribution à d'autres Objectifs d'Aichi le cas échéant

Ce n'est pas une mesure pour l'Objectif 11. Évaluer la contribution à d'autres Objectifs d'Aichi le cas échéant 
Signaler en tant qu'aire protégée pour l'Objectif 11 et évaluer sa contribution à d'autres Objectifs le cas échéant

La mesure atteint son objectif en conservant les écosystèmes et les habitats, et en maintenant et/ou reconstituant des populations viables d'espèces dans leur milieu naturel

La mesure n'atteint pas son objectif en conservant les écosystèmes et les habitats, et en maintenant et/ou reconstituant des populations viables d'espèces dans leur milieu naturel
Évaluer en tant qu'AMCE potentielle pour l'Objectif 11 et la contribution à d'autres Objectifs le cas échéant

Évaluer en tant qu'AMCE potentielle pour l'Objectif 11 et la contribution aux Objectifs 12 et 13 , et à d'autres le cas échéant

Ce n'est pas une mesure pour l'Objectif 11 . Évaluer la contribution aux Objectifs 12 et 13 , et à d'autres le cas échéant

Évaluer en tant qu'AMCE potentielle pour l'Objectif 11 et la contribution aux Objectifs 5, 6, 7 et 10 , et à d'autres le cas échéant

Ce n'est pas une mesure pour l'Objectif 11 .

Évaluer la contribution aux Objectifs 5, 6, 7 et 10, et à d'autres le cas échéant
La mesure atteint son objectif en conservant les écosystèmes et les habitats, et en maintenant et/ou reconstituant des populations viables d'espèces dans leur milieu naturel

La mesure n'atteint pas son objectif en conservant les écosystèmes et les habitats, et en maintenant et/ou reconstituant des populations viables d'espèces dans leur milieu naturel
Évaluer en tant qu'AMCE potentielle pour l'Objectif 11 et la contribution aux Objectifs 14 et 15 , et à d'autres le cas échéant

Ce n'est pas une mesure pour l'Objectif 11. Évaluer la contribution aux Objectifs 14 et 15, et à d'autres le cas échéant

$\begin{aligned} & \text { LÉGENDE } \\ & \longrightarrow \text { En vert : Chemin primaire pour considération en tant } \\ & \text { que mesure potentielle pour l'Objectif } 11\end{aligned}$




\section{Annexe III}

\section{Base de données mondiale sur les aires protégées}

\section{Bases de données de Protected Planet sur les aires protégées et les AMCE}

All data on other effective area-based conservation measures (OECMs/conserved areas) should be submitted to the UN Environment World Conservation Monitoring Centre (UNEP-WCMC) to be added to the World Database on Protected Areas (WDPA).

Des orientations complémentaires sont disponibles sur ce lien : www.wcmc.io/conservedareas_guidance

\section{Qu'est-ce que la Base de données mondiale sur les aires protégées (WDPA) ?}

La WDPA est la base de données mondiale la plus complète sur les aires protégées marines et terrestres, comprenant à la fois des données spatiales (c.-à-d. frontières et points) et des données d'attributs connexes (c.-à-d. des informations sous forme de tableaux), recueillies de manière normalisée. Les informations « source " sont également conservées pour tous les ensembles de données soumis. La WDPA est actualisée tous les mois et est disponible et téléchargeable à partir du site Protected Planet, à l'exception des données qui ont des restrictions de partage imposées par les fournisseurs de ces données. Le Manuel de l'utilisateur de la WDPA (UNEP-WCMC, 2017) fournit des informations détaillées et des orientations sur les données figurant dans la WDPA, y compris leur consolidation et leur normalisation. La nouvelle base de données des AMCE suit la même structure que la WDPA, avec de légères modifications. La WDPA et la base de données des AMCE sont les sources de données officielles utilisées par plusieurs mécanismes d'établissement de rapports au niveau mondial, notamment pour les indicateurs et le suivi de l'avancement par rapport aux objectifs des aires protégées et conservées, y compris dans le cadre des Objectifs d'Aichi pour la biodiversité du Plan stratégique de la CDB et des Objectifs de développement durable (ODD) des Nations Unies.

\section{Rapports, collecte de données et validation des aires protégées et des AMCE}

Généralement, les données sont soumises à la WDPA ou à la base de données des AMCE par l'autorité de gouvernance de l'aire protégée ou conservée, et ces données sont prioritaires par rapport aux données soumises par d'autres sources concernant la même zone. Lorsque l'autorité de gouvernance n'est pas en mesure de fournir une actualisation par manque de capacité ou de données, ou pour d'autres raisons, elle peut suggérer qu'un autre fournisseur de données soit contacté pour une mise à jour. Tous les sites doivent répondre à la définition d'une aire protégée ou d'une " autre mesure de conservation efficace par zone » fournie par l'UICN/la CDB.

Une seule version de toute aire protégée ou conservée est stockée dans les bases de données de Protected Planet. Lorsque des zones se chevauchent, il s'agit généralement de désignations différentes qui couvrent le même espace géographique. Toutes les données de la WDPA ou de la base de données des AMCE doivent répondre à un ensemble de normes relatives aux données. Les normes sont importantes pour garantir que toutes les informations sont fournies selon un format commun qui est interopérable et utile pour de nombreux types de rapports et d'analyses. Quatre exigences clés doivent être respectées pour se conformer aux normes de Protected Planet relatives aux données :

1. Tous les sites doivent répondre à la définition d'une aire protégée ou d'une « autre mesure de conservation efficace par zone » fournie par I'UICN/la CDB.

2. Les données spatiales des Systèmes d'informations géographiques (SIG) et une liste correspondante d'attributs normalisés doivent être fournies.

3. La source des informations doit être fournie pour garantir que la propriété des données est préservée et traçable.

4. Un Contrat de fournisseur de données doit être signé pour s'assurer qu'il existe une trace écrite confirmant l'accord du fournisseur de données au sujet de l'intégration des données dans la WDPA ou la base de données des AMCE, et des conditions de leur mise à disposition.

Utilisation des bases de données de Protected Planet pour mesurer les progrès par rapport aux Objectifs

Le PNUE-WCMC utilise les données figurant dans les bases de données de Protected Planet pour mesurer les progrès enregistrés par rapport aux objectifs de conservation internationaux, comme l'Objectif 11 d'Aichi pour la biodiversité. Pour l'établissement de rapports relatifs à l'Objectif 11, trois statistiques seront générées (au niveau national, régional et mondial) :

- Couverture des aires protégées ;

- Couverture des autres mesures de conservation efficaces par zone ; et

- Couverture cumulée

Pour calculer la couverture, le PNUE-WCMC supprime les chevauchements entre les sites et exclut certaines catégories de sites (c.-à-d. les sites proposés, les points sans zone signalée et les réserves de biosphère MAB de l'UNESCO). Même si les aires conservées et les aires protégées n'occupent généralement pas la même zone (voir Section 3.2 b), il peut exister des cas occasionnels de chevauchement. Dans de tels cas, la zone 
de chevauchement est traitée comme une aire protégée uniquement. Cette méthode évite la double comptabilisation. De plus amples informations sur la façon dont le PNUE-WCMC calcule les statistiques de couverture sont disponibles sur https:// protectedplanet.net/c/calculating-protected-area-coverage

\section{Suivi des autres mesures de conservation efficaces par zone}

L'Efficacité de la gestion des aires protégées (EGAP) sera, dans de nombreux cas, le moyen le plus pragmatique de mesurer l'efficacité des aires protégées, en particulier lorsque les outils d'EGAP s'appuient sur des informations supplémentaires concernant les résultats pour la biodiversité. Plus de 40 outils ont été créés pour les évaluations de l'EGAP (voir UNEP-WCMC, 2018). Avec l'adoption des systèmes d'EGAP existants, il sera plus facile pour l'autorité d'établir un rapport de suivi de l'efficacité pour le PNUE-WCMC, et les évaluations seront dans un format normalisé entre les sites et au fil du temps.

Des principes de base pour le suivi des aires conservées, afin de surveiller l'efficacité de la conservation, sont décrits aux étapes 1 à 4 ci-dessous. Les étapes 1 à 3 peuvent aussi être utilisées pour décider si un site est une aire conservée, ou bien s'il est toujours conservé efficacement après des évaluations répétées.

5. Décrire toutes les valeurs importantes de la biodiversité sur le site, en enregistrant les sources d'information à l'appui. Prendre en compte la représentativité, l'intégrité, le contexte du paysage terrestre, les habitats et espèces rares, menacés, endémiques et importants, et l'intégrité écologique.

6. Identifier les pressions et les menaces pesant sur le site, qui auront des répercussions sur les valeurs de biodiversité.

7. Examiner les interventions et mesures de gestion réalisées sur le site, pour déterminer si elles : sont efficaces et suffisantes pour préserver les caractéristiques de la biodiversité, couvrent l'intégralité de la biodiversité présente sur le site, et répondent aux menaces contrôlables pesant sur la conservation in situ de la biodiversité.

8. Examiner l'efficacité en termes de résultats pour la conservation sur le site, en mesurant l'état des attributs prioritaires, en établissant et en examinant des objectifs et indicateurs qui mesurent l'état et les tendances au fil du temps, en mesurant l'atténuation des menaces, en effectuant un suivi et en gérant de façon adaptative.

L'établissement de rapports destinés à la Base de données mondiale sur l'efficacité de la gestion des aires protégées (GD-PAME), gérée par le PNUE-WCMC, suit une approche similaire à celle décrite ci-dessus pour la WDPA et la base de données des AMCE. Pour toute question concernant l'établissement de rapports, la consolidation, l'utilisation ou le traitement de la GD-PAME, veuillez contacter : 




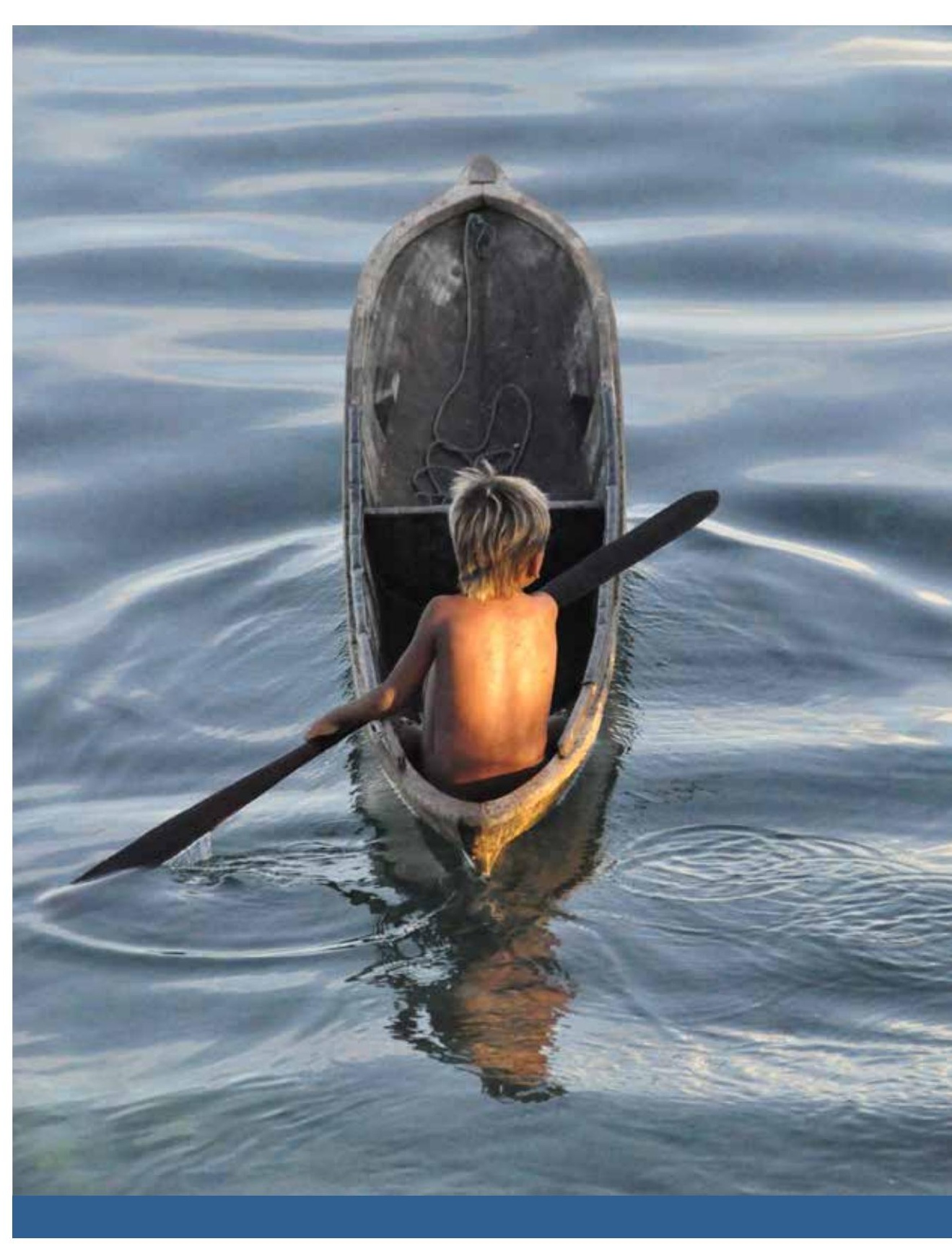

UNION INTERNATIONALE POUR LA CONSERVATION DE LA NATURE

SIÈGE MONDIAL

Rue Mauverney 28

1196, Gland, Suisse

Tel: +41 229990000

Fax: +41229990002

www.iucn.org/fr 